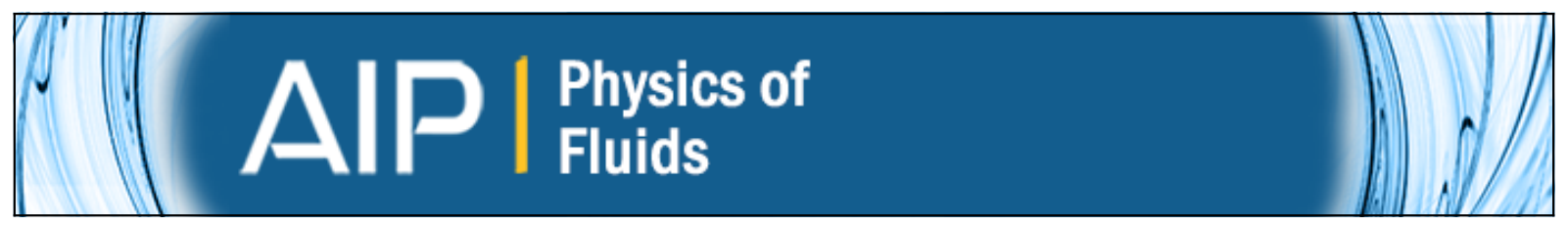

\title{
A lift formula applied to low-Reynolds-number unsteady flows
}

Shizhao Wang, Xing Zhang, Guowei He, and Tianshu Liu

Citation: Physics of Fluids (1994-present) 25, 093605 (2013); doi: 10.1063/1.4821520

View online: $\mathrm{http} / / / \mathrm{dx}$.doi.org/10.1063/1.4821520

View Table of Contents: http://scitation.aip.org/content/aip/journal/pof2/25/9?ver=pdfcov

Published by the AIP Publishing

\section{AlP Re-register for Table of Content Alerts}




\title{
A lift formula applied to low-Reynolds-number unsteady flows
}

\author{
Shizhao Wang, ${ }^{1}$ Xing Zhang, ${ }^{1}$ Guowei He, ${ }^{1, \text { a) }}$ and Tianshu Liu ${ }^{1,2}$ \\ ${ }^{1}$ The State Key Laboratory of Nonlinear Mechanics, Institute of Mechanics, \\ Chinese Academy of Sciences, Beijing 100190, People's Republic of China \\ ${ }^{2}$ Department of Mechanical and Aeronautical Engineering, Western Michigan University, \\ Kalamazoo, Michigan 49008, USA
}

(Received 10 December 2012; accepted 29 August 2013;

published online 23 September 2013)

A lift formula for a wing in a rectangular control volume is given in a very simple and physically lucid form, providing a rational foundation for calculation of the lift of a flapping wing in highly unsteady and separated flows at low Reynolds numbers. Direct numerical simulations on the stationary and flapping two-dimensional flat plate and rectangular flat-plate wing are conducted to assess the accuracy of the lift formula along with the classical Kutta-Joukowski theorem. In particular, the Lamb vector integral for the vortex force and the acceleration term of fluid for the unsteady inertial effect are evaluated as the main contributions to the unsteady lift generation of a flapping wing. ( 2013 AIP Publishing LLC. [http://dx.doi.org/10.1063/1.4821520]

\section{INTRODUCTION}

Low-Reynolds-number flight particularly flapping flight has recently attracted considerable attention in the aeronautical communities due to the need of developing biologically inspired micro air vehicles (MAV) ${ }^{1,2}$ Therefore, animal flight, which has been traditionally studied by avian zoologists, becomes immediately relevant to this engineering research. Natural flyers include birds, insects, and bats. In bird flight, the Reynolds numbers based on the mean wing chord range from $10^{4}$ to $10^{6}$ while the Reynolds numbers for bat flight are $10^{3}-10^{5}$. In contrast, the Reynolds numbers for insects are typically less than 5000. To understand unsteady flow fields around flying birds and bats, particle image velocimetry measurements have been conducted in wind tunnels. ${ }^{3-7}$ Since the aerodynamic forces of a flying animal cannot be directly measured using a force balance, the Kutta-Joukowski (K-J) theorem has been used in these studies to infer the lift after the circulation is estimated by integrating the vorticity field in a selected cross-section region near wings or in wakes generated in flapping flight. The advantage of using the K-J theorem is its simplicity, which is directly related to vorticty fields that can be measured by PIV. In particularly, it allows estimation of the lift components contributed by certain distinct vortical structures such as the leading-edge vortices. On the other hand, to calculate the lift, the K-J theorem has served as an essential mechanism in vortex-based aerodynamics models for low-Reynolds-number flapping flight. ${ }^{3,8-12}$ The classical aerodynamics models have been adapted further by incorporating some relevant flow phenomena like the leading- and trailing-edge vortices for additional lift generation. ${ }^{13-15}$

However, lift estimation using the K-J theorem based on PIV measurements in the wakes of slowly flying birds (pigeons and jackdaws) gave a significantly lower value of the lift that cannot support the bird weight. It is known as "the wake momentum paradox." ${ }^{3}$ Recent PIV measurements in the wakes of flying bats by Hubel et al. ${ }^{6,7}$ also indicated that the lift estimated by using the

\footnotetext{
a) Author to whom correspondence should be addressed. Electronic mail: hgw@lnm.imech.ac.cn. Telephone: 86-1082543969 .
} 
K-J theorem is too low to support the bat weight at low flight speeds. It has been shown that the K-J theorem is not satisfactory for lift estimation in unsteady and separated flows at low Reynolds numbers although it has been popularly used in the field of biolocomotions. Therefore, it is highly desirable to derive a lift formula to replace the K-J theorem in this field, which motivates the present work.

The K-J theorem is probably the earliest and simplest expression directly relating the fluidmechanic force to the velocity-related quantities. The general force expressions have been comprehensively discussed by Saffman ${ }^{16}$ mainly in the framework of inviscid flows. From the Navier-Stokes (NS) equations, various forms of the force expressions have been derived depending on how to transform the pressure term to the velocity-related quantities. ${ }^{17-23}$ In principle, a general lift formula can be derived by directly projecting these general force expressions onto the direction normal to the incoming flow. However, in this way, a compact form of the general lift formula has not been achieved since these force expressions usually contains many terms as a consequence of eliminating the pressure term.

The objective of this study is to give a simple but sufficiently accurate lift formula to replace the K-J theorem for estimation of the lift in highly unsteady and separated flows associated with flapping flight at low Reynolds numbers. The accuracy of the lift formula is quantitatively evaluated through direct comparison with the lift calculated from direct numerical simulations (DNS) on low-Reynolds-number flows over the stationary and flapping two-dimensional (2D) flat plate and rectangular flat-plate wing. This paper is organized as follows. The lift formula is derived from the NS equations for a rectangular control volume. Since the K-J theorem is just a reduced case of the lift formula, the mechanisms neglected in the K-J theorem applied to unsteady flows are identified. The numerical method, the immersed boundary method based on discrete stream function formulation, and its validation are briefly described. Then, the flows over the stationary and flapping flat plate are simulated, and the lift coefficients given by using the lift formula are directly compared with DNS. Finally, the flows over the stationary and flapping rectangular flat-plate wing with the aspect ratio of 4 are simulated to further examine the accuracy of the lift formula in three-dimensional (3D) cases. The advantages of the lift formula and the limitations of the K-J theorem applied to these flows are discussed. The physical meanings of the inertial terms in the lift formula are further elucidated in Appendix A and the examples of code validation are given in Appendix B.

\section{FORCE EXPRESSIONS}

\section{A. Fluid-mechanic force}

In classical aerodynamics, the K-J theorem is derived in the 2D potential flow over a circular cylinder with a given value of the circulation, and then it is extended to an airfoil via a conformal transformation where the circulation is determined by applying the Kutta condition at the trailing edge. ${ }^{24,25}$ Further, in the Prandtl lifting-line theory, the K-J theorem is applied to a bounded vortex line along the wing span to model the aerodynamic flow over a finite wing. Although the K-J theorem is originally derived in the framework of the inviscid flow theory, the circulation is physically originated from the viscous shearing motion in a boundary layer around an airfoil. Actually, the Kutta condition represents the outcome of the generation of the circulation through viscous shearing. The application of the K-J theorem is well founded for high-Reynolds-number attached flows over airfoils since the flow outside a thin boundary layer can be considered to be inviscid. The circulation can be calculated by integrating the vorticity field in a boundary layer in a suitably large integration domain covering the upper and lower surfaces of an airfoil. However, in flight of small birds, bats, and insects, not only the Reynolds numbers are usually much smaller but also the flow around a flapping thin wing at high angle of attack (AoA) is highly unsteady and separated, generating energetic and organized vortices such as the leading- and trailing-edge vortices. In fact, the lift generated by these vortices in unsteady separated flows is necessary for low-Reynolds number animal flight. In this case, the legitimacy of applying the K-J theorem has to be examined in the framework of the viscous flow theory. 


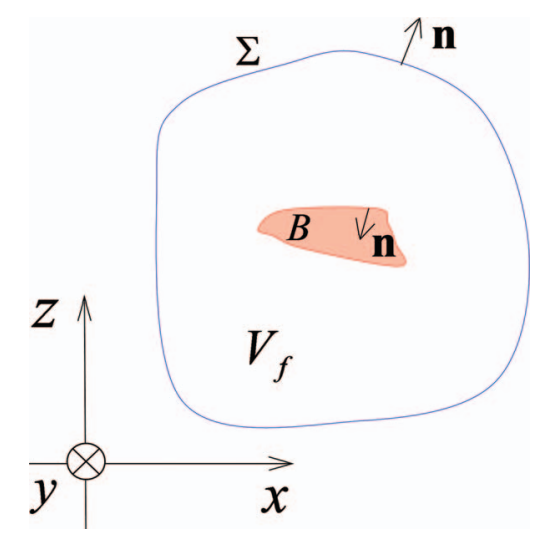

FIG. 1. Schematic of a control surface around a body.

In an incompressible viscous flow, as shown in Figure 1, the force acting on a solid body is given by

$$
\boldsymbol{F}=-\oint_{\partial B}(-p \boldsymbol{n}+\boldsymbol{\tau}) d S=-\rho \int_{V_{f}} \boldsymbol{a} d V+\oint_{\Sigma}(-p \boldsymbol{n}+\boldsymbol{\tau}) d S
$$

where $p$ is the pressure, $\boldsymbol{\tau}$ is the surface shear stress vector, $\boldsymbol{a}=D \boldsymbol{u} / D t$ is the acceleration, $\rho$ is the fluid density, $\partial B$ denotes a solid boundary of the body domain $B, V_{f}$ denotes the control volume of fluid, $\Sigma$ denotes an outer control surface in which the body is enclosed, and $\boldsymbol{n}$ is the unit normal vector pointing to the outside of a control surface. It is difficult to use Eq. (1) to infer the force in measurements. It is not easy to measure surface pressure and skin friction fields in low-speed flows even though pressure-sensitive paint measurement and global skin friction diagnostics are promising to obtain the integrated forces. ${ }^{26,27}$ Therefore, a simple alternative force formula is desirable.

Substitution of $\boldsymbol{a}=D \boldsymbol{u} / D t=\partial \boldsymbol{u} / \partial t+\boldsymbol{u} \cdot \nabla \boldsymbol{u}=\partial \boldsymbol{u} / \partial t+\boldsymbol{\omega} \times \boldsymbol{u}+\nabla\left(q^{2} / 2\right)$ into Eq. (1) leads to

$$
\boldsymbol{F}=\rho \int_{V_{f}} \boldsymbol{u} \times \boldsymbol{\omega} d V-\rho \int_{V_{f}} \frac{\partial \boldsymbol{u}}{\partial t} d V-\oint_{\Sigma}\left(p+\rho q^{2} / 2\right) \boldsymbol{n} d S+\oint_{\Sigma} \boldsymbol{\tau} d S-\rho \oint_{\partial B}\left(q^{2} / 2\right) \boldsymbol{n} d S,
$$

where $\boldsymbol{u}$ is the velocity, $\boldsymbol{\omega}$ is the vorticity, and $q=|\boldsymbol{u}|$. The first term in the right-hand side (RHS) of Eq. (2) is a volume integral of the Lamb vector $\boldsymbol{u} \times \boldsymbol{\omega}$ that represents the vortex force. ${ }^{16}$ For convenience, it is simply called the Lamb vector integral. The second term is a volume integral of the local acceleration of fluid induced by a moving solid body for the unsteady inertial effect. The third and fourth terms are the surface integrals of the total pressure $P=p+\rho q^{2} / 2$ and the surface shear stress on the control surface $\Sigma$. The fifth term is the boundary term. In an inviscid irrotational unsteady flow where the first, third, and fourth terms in Eq. (2) vanish, the remaining second and fifth terms together are interpreted as the added mass force in ideal fluid mechanics. The fifth term can be written as $\rho V_{B}\langle q \nabla q\rangle_{B}$ by using Gauss' theorem, where $V_{B}$ is the volume of the body and $\langle\bullet\rangle_{B}$ is a domain-average operator defined as $\langle\bullet\rangle_{B}=V_{B}^{-1} \int_{B} \bullet d V$. It is indicated in Appendix A that for a thin wing the fifth term is small which is interpreted as the part of the added mass force associated with the fluid virtually occupying the body domain $B$ (a virtual fluid body). The physical meanings of the second and fifth terms are further discussed in Appendix A.

Since the static pressure $p$ in space is very difficult to measure, the third term related to $p$ in Eq. (2) should be transformed to the terms related to the velocity that is measurable. Different approaches have been used to deal with the troublesome pressure term, which leads to various force expressions. ${ }^{17-23}$ However, for a general control surface, eliminating the pressure term usually results in more complicated expressions in which the physical meanings and relative contributions of some terms cannot be easily elucidated. By introducing an auxiliary velocity potential satisfying suitable boundary conditions and projecting the NS equations on the gradient of the potential, Quartapelle and Napolitano, ${ }^{28}$ Howe,${ }^{18}$ and Chang ${ }^{17}$ were able to extract the explicit pressure force from 


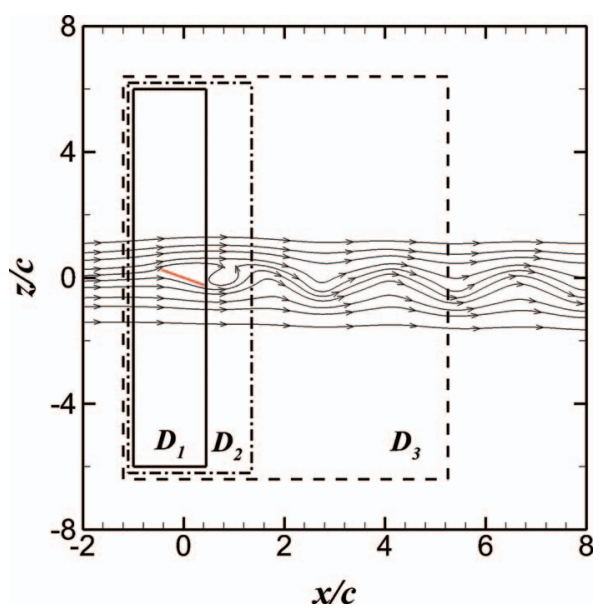

FIG. 2. Integral domains enclosing an airfoil or a wing.

the pressure term and decompose the force into several terms whose physical meanings become clear. Although these types of the expressions are valuable from a theoretical viewpoint, they are not easy to use for processing experimental data since the potential cannot be measured. The expressions based on "derivative-moment transformations" of Wu et al. ${ }^{22}$ provide another way to investigate the role of local flow structures on the force and moment.

\section{B. Lift formula}

In this work, we circumvent this pressure problem by selecting a rectangular control volume to obtain a very simple lift formula. Consider a coordinate system $(x, y, z)$ defined by the unit orthogonal vectors $(\boldsymbol{i}, \boldsymbol{j}, \boldsymbol{k})$ where $\boldsymbol{i}$ and $\boldsymbol{k}$ are the unit vectors parallel and normal to the freestream, respectively, and $\boldsymbol{j}=\boldsymbol{k} \times \boldsymbol{i}$ is the unit vector along the spanwise direction. The lift on a body is given by $L=F_{z}=\boldsymbol{k} \cdot \boldsymbol{F}$. More specifically, as shown in Figure 2, a rectangular domain $D$ is selected as a control volume to simplify the lift expression. The unit normal vectors on the left and right faces (sections) of the boundary $\partial D$ are perpendicular to $\boldsymbol{k}$, i.e., $\boldsymbol{k} \cdot \boldsymbol{n}=0$. Note that for 3D flows the unit vectors on the side faces of the control volume are also perpendicular to $\boldsymbol{k}$, i.e., $\boldsymbol{k} \cdot \boldsymbol{n}=0$. Furthermore, if the top and bottom sections of $\partial D$ are located so far away from the body that the steady-state potential flow in forward flight is reached (i.e., $P=p+\rho q^{2} / 2 \rightarrow$ const. according to the Bernoulli equation), the integrals on these sections are cancelled out. In this case, the contribution of the troublesome third term (the total pressure term) of RHS of Eq. (2) to the lift is zero. However, on a limited domain boundary $\partial D$ where the inviscid outer flow could be disturbed by induction of unsteady vortical structures, the contribution of the total pressure term to the lift does not completely vanish. The error associated with this perturbation will be discussed later. Furthermore, for a sufficiently large boundary $\partial D$, the viscous stress term $\boldsymbol{k} \cdot \boldsymbol{\tau}$ on the most portion of $\partial D$ is zero except in a wake, and thus the contribution of the fourth term in RHS of Eq. (2) to the lift is very small. The fifth term in RHS of Eq. (2), $\rho V_{B}\langle q \nabla q\rangle_{B}$, can be readily evaluated when the kinematics of a body is given.

After the total pressure and viscous stress terms on the outer boundary $\Sigma$ are neglected, the contributions of the other terms in RHS of Eq. (2) are retained, and as a result the lift is given by a very simple formula,

$$
L=\rho V_{D} \boldsymbol{k} \cdot\left(\langle\boldsymbol{u} \times \boldsymbol{\omega}\rangle_{D}-\langle\partial \boldsymbol{u} / \partial t\rangle_{D}\right)+\rho V_{B} \boldsymbol{k} \cdot\langle q \nabla q\rangle_{B},
$$

where $\langle\bullet\rangle_{D}$ is the domain-averaged operator $\langle\bullet\rangle_{D}=D^{-1} \int_{D} \bullet d V$ and $D$ is a rectangular domain. Compared to the K-J theorem, Eq. (3) is a more general lift formula which contains the two leadingorder terms: the Lamb vector integral for the vortex force and the local vertical acceleration for the unsteady inertial effect. For a thin wing, the term $\rho V_{B}\langle q \nabla q\rangle_{B}$ is negligibly small compared to the 
first term in Eq. (3). The form of the Lamb vector integral and the local vertical acceleration allows domain decomposition in which they can be expressed as a sum of the corresponding terms in subdomains. This is advantageous since the contributions of individual flow structures in sub-domains to the lift can be directly distinguished and evaluated.

In two dimensions, the sectional lift is given by

$$
L^{\prime}=\rho D\left(\left\langle u_{x} \omega_{y}\right\rangle_{D}-\left\langle\partial u_{z} / \partial t\right\rangle_{D}\right)+\rho V_{B}\langle q \partial q / \partial z\rangle_{B},
$$

where $\boldsymbol{u}=\left(u_{x}, u_{z}\right)$ is the fluid velocity vector, the $x$ - and $z$-coordinates are in the freestream and vertical directions, respectively, $D$ denotes the domain area in two dimensions, and $\langle\bullet\rangle_{D}$ becomes the area-averaged operator in 2D. Rigorously speaking, the lift formula, Eq. (3) or (4), is valid only for a rectangular control volume, which seems a considerably constrained case in theory. Nevertheless, this does not limit the usefulness of Eq. (3) or (4) because a simple rectangular domain is often used anyway for data processing in experiments and computations. Equation (4) is particularly suitable for lift estimation in experiments since the two velocity components $\left(u_{x}, u_{z}\right)$ on a streamwise crosssection and the spanwise vorticity $\omega_{y}$ are routinely measured using planar PIV in wind and water tunnels.

To compare Eq. (4) with the K-J theorem, the local effective velocity (the vorticity-weighted velocity) is defined as $u_{e f f}=u_{x} \omega_{y} /\left\langle\omega_{y}\right\rangle_{D}$, where $\left\langle\omega_{y}\right\rangle_{D}$ is the area-averaged vorticity. Further, by introducing the area-averaged effective velocity $U_{\text {eff }}=\left\langle u_{\text {eff }}\right\rangle_{D}$, Eq. (4) can be written in a form analogous to the K-J theorem

$$
L^{\prime}=\rho U_{e f f} \Gamma-\rho D\left\langle\partial u_{z} / \partial t\right\rangle_{D}+\rho V_{B}\langle q \partial q / \partial z\rangle_{B},
$$

where $\Gamma=\left\langle\omega_{y}\right\rangle_{D} D$ is the circulation. Unlike the classical K-J theorem $L_{K-J}^{\prime}=\rho U_{\infty} \Gamma$, the domainaveraged effective velocity $U_{\text {eff }}$ rather than $U_{\infty}$ is used in Eq. (5), which depends on not only the velocity and vorticity distributions around a body but also time in general. In addition, $-\rho D\left\langle\partial u_{z} / \partial t\right\rangle_{D}+$ $\rho V_{B}\langle q \partial q / \partial z\rangle_{B}$ explicitly represents the unsteady inertial effect that is omitted by the K-J theorem. For unsteady flows, application of the K-J theorem implies the quasi-steady-state assumption in which $L_{K-J}^{\prime}(t)$ is synchronized with $\Gamma(t)$. This assumption is generally problematic, which could lead to errors in the time-averaged magnitude and phase of the unsteady lift. To illustrate this point, by introducing a velocity difference $v(t)=U_{e f f}(t)-U_{\infty}$ that represents a certain perturbation velocity, Eq. (5) gives $L^{\prime}(t)-L_{K-J}^{\prime}(t)=\rho v(t) \Gamma(t)-\rho D\left\langle\partial u_{z} / \partial t\right\rangle_{D}+\rho V_{B}\langle q \partial q / \partial z\rangle_{B}$. Clearly, it is indicated that $L^{\prime}(t)$ and $L_{K-J}^{\prime}(t)$ could considerably differ in the phase, amplitude, and waveform of the unsteady lift particularly when the velocity fluctuation $v(t)$ and the acceleration term are large. The relation for the time-averaged lift is $\overline{L^{\prime}}-{\overline{L^{\prime}}}_{K-J}=\rho \overline{v \Gamma}-\rho D\left\langle\overline{\partial u_{z} / \partial t}\right\rangle_{D}+\rho V_{B}\langle\overline{q \partial q / \partial z}\rangle_{B}$, where the bar - denotes the time-average.

In the above analysis, the contribution of the total pressure term in Eq. (2) to the lift vanishes when the top and bottom boundaries of a sufficiently large rectangular domain reach to the steady-state potential flow. However, the potential flow is disturbed by induction of unsteady vortical structures generated by flow separation or a moving body. On the top and bottom boundaries of a relatively small domain, the total pressure is a function of time, i.e., $p+\rho q^{2} / 2=$ const. $+F(t)-\rho \partial \phi / \partial t$, where $\phi$ is the velocity potential, and $\partial \phi / \partial t$ and $F(t)$ depend on the boundary conditions. In this case, the total pressure term in Eq. (2) equals to the surface integral of the difference of the perturbation term $F(t)-\rho \partial \phi / \partial t$ between the top and bottom boundaries. Based on an analysis of the potential flow perturbed by point vortices, the surface integral of this difference of the perturbation term decays in a fashion of $O(c / Y)$, where $c$ is the chord and $Y$ is the vertical distance of the upper boundary from the body. For a finite domain, this error should be estimated by numerical computations for typical flows. The contribution of the total pressure term is evaluated quantitatively through DNS for a stationary and flapping flat plate in Sec. IV. In computations, the top and bottom boundaries of a rectangular domain are located at $z / c= \pm 6$, where $c$ is the chord of the plate. It is found that the total pressure term fluctuates at the same frequency as the unsteady lift with a certain phase shift, indicating that the error may be indeed caused by the perturbation induced by the unsteady vortical structures. The relative error in the time-averaged lift due to neglecting the total pressure term is less than $2 \%$. In general, numerical simulations indicate that the contributions of the top and bottom boundaries to the lift can be neglected when $d /(c \sin \alpha) \sim O(10)$ for the stationary plate and 
$d /(2 A) \sim O(10)$ for the flapping plate at the flapping Strouhal number of 0.3 are selected, where $d$ is the distance between the top/bottom boundary and the body, $c$ is the chord, $\alpha$ is the angle of attack, and $A$ is the flapping amplitude. Therefore, it is legitimate to ignore the contribution of the total pressure term in Eq. (2) to the lift generation when a rectangular domain is sufficiently large.

\section{The Kutta-Joukowski theorem}

The classical K-J theorem is a reduced form of Eq. (5). In a steady 2D flow where $\left\langle\partial u_{z} / \partial t\right\rangle_{D}=$ 0 and $V_{B}\langle q \partial q / \partial z\rangle_{B}=0$, a vorticity patch bounded in a finite region that is much smaller than the rectangular control domain $D$ is considered. The $x$-component velocity can be decomposed into $u_{x}$ $=U_{\infty}+u_{x}^{\prime}$, where $u_{x}^{\prime}$ is the velocity induced by the vorticity patch along the $x$-coordinate that is given by

$$
u_{x}^{\prime}(x, z)=\frac{1}{2 \pi} \iint_{D} \frac{(z-\eta) \omega_{y}(\xi, \eta)}{(x-\xi)^{2}+(z-\eta)^{2}} d \xi d \eta .
$$

Therefore, since $u_{e f f}=u_{x} \omega_{y} /\left\langle\omega_{y}\right\rangle_{D}=U_{\infty} \omega_{y} /\left\langle\omega_{y}\right\rangle_{D}+u_{x}^{\prime} \omega_{y} /\left\langle\omega_{y}\right\rangle_{D}$, the domain-averaged effective velocity is

$$
U_{e f f}=\left\langle u_{e f f}\right\rangle_{D}=U_{\infty}+\frac{1}{2 \pi} \frac{1}{\left\langle\omega_{y}\right\rangle_{D} D} \iiint \int \frac{(z-\eta) \omega_{y}(\xi, \eta) \omega_{y}(x, z)}{(x-\xi)^{2}+(z-\eta)^{2}} d \xi d \eta d x d z
$$

where the integration domain in both $(\xi, \eta)$ and $(x, z)$ is $D$. When the variables $(\xi, \eta)$ and $(x, z)$ are interchanged, the factor $z-\eta$ in Eq. (7) changes its sign. It is known that the integral in Eq. (7) must vanish such that $U_{\text {eff }}=U_{\infty}$. In this case, the K-J theorem $L_{K-J}^{\prime}(t)=\rho U_{\infty} \Gamma$ is exactly recovered from Eq. (5). The above deduction is similar to that given by von Karman and Burgers ${ }^{29}$ for a bundle of vortex lines. The derivation of the K-J theorem from a general theory of viscous flows has been also given by $\mathrm{Wu},{ }^{30,31} \mathrm{Wu}$ et al., ${ }^{21}$ and Schmitz and Chattot. ${ }^{32}$

\section{Drag}

A sufficiently large rectangular domain $D$ is considered as shown in Figure 2. Similarly, by neglecting the small terms, the force in the freestream direction (drag plus thrust) on a body is given by

$$
F_{x}=\boldsymbol{i} \cdot \boldsymbol{F}=\rho \boldsymbol{i} \cdot \int_{V_{f}} \boldsymbol{u} \times \boldsymbol{\omega} d V-\rho \boldsymbol{i} \cdot \int_{V_{f}} \frac{\partial \boldsymbol{u}}{\partial t} d V-\boldsymbol{i} \cdot \oint_{\Sigma}\left(p+\rho q^{2} / 2\right) \boldsymbol{n} d S,
$$

where $\boldsymbol{i}$ is the unit vector parallel to the freestream. The first term in RHS of Eq. (8) is the contribution of the vortex force that physically contains the induced drag and the thrust generated by a flapping wing. The second term is the contribution of the local acceleration in the freestream direction in unsteady flows. For a thin wing, the fifth term in Eq. (2) is neglected. However, unlike the case of lift, for a sufficiently large rectangular domain, the total pressure term (the third term) in RHS of Eq. (8) cannot be neglected, and it can be expressed by an integral over a transverse plane $S_{1}$

$$
D_{p}=\int_{S_{1}}\left[\rho\left(q_{\infty}^{2}-q_{1}^{2}\right) / 2+\left(p_{\infty}-p_{1}\right)\right] d S .
$$

Physically, this term represents the parasite drag. Since a pressure field is very difficult to measure, it is preferred to transform the total pressure term in RHS of Eq. (9) to an expression based on a velocity filed. A useful relation obtained from the NS equations is ${ }^{20}$

$$
\oint_{\Sigma}\left(p+\rho q^{2} / 2\right) \boldsymbol{n} d S=\frac{\rho}{N-1} \oint_{\Sigma} \boldsymbol{x} \times \boldsymbol{n} \times \boldsymbol{v}_{p} d S
$$

where $\boldsymbol{v}_{p}=\partial \boldsymbol{u} / \partial t-\boldsymbol{u} \times \boldsymbol{\omega}-v \nabla^{2} \boldsymbol{u}$ and the dimensionality is defined as $N=3$ for 3D or $N=2$ for $2 \mathrm{D}$. Therefore, the drag is expressed in terms of the measurable quantities $\boldsymbol{u}, \partial \boldsymbol{u} / \partial t$, and $\boldsymbol{\omega}$. For a sufficiently large control surface where flow can be considered to be inviscid, the viscous diffusion term can be neglected. 


\section{NUMERICAL METHOD AND SETTING}

The 2D and 3D flows around a flat plate are simulated using an immersed boundary method based on discrete stream function formulation developed by Wang and Zhang. ${ }^{33}$ The incompressible Navier-Stokes equations with additional body forces and the continuity equation are used as the governing equations

$$
\begin{gathered}
\frac{\partial \boldsymbol{u}}{\partial t}+\boldsymbol{u} \cdot \nabla \boldsymbol{u}=-\nabla p+\frac{1}{\operatorname{Re}_{c}} \nabla^{2} \boldsymbol{u}+f, \\
\nabla \cdot \boldsymbol{u}=0,
\end{gathered}
$$

where $\boldsymbol{u}$ is the non-dimensional velocity, $p$ is the non-dimensional pressure, and $\boldsymbol{f}$ is the nondimensional body forces representing the effects of the boundaries. The Reynolds number is defined as $\operatorname{Re}_{c}=U c / v$, where $U$ is the upstream flow velocity, $c$ the chord length, and $v$ the kinematic viscosity. Equations (11) and (12) are solved on an unstructured Cartesian (Eulerian) mesh in the framework of discrete stream function formulation. The plate is marked using a set of Lagrangian points. The Eulerian mesh and Lagrangian points are coupled using the interpolation based on discrete $\delta$ function. The discrete form of body force $f$ is determined implicitly by solving a linear system to implement the non-slip boundary conditions on the surface of the plate.

The simulations are conducted in a domain of $[-12,20] \times[-16,16]$ in streamwise $(x)$ and vertical $(z)$ directions for 2D flows, and $[-12,20] \times[-16,16] \times[-16,16]$ in streamwise $(x)$, spanwise (y), and vertical (z) directions for 3D flows. The center of the plate is positioned at the origin. The unstructured mesh with hanging-node is used in the simulations to refine the mesh around the plate. The minimum grid size used for the $2 \mathrm{D}$ and $3 \mathrm{D}$ flows are $0.01 \mathrm{c}$ and $0.02 \mathrm{c}$, respectively. The time step is selected to keep the maximum CFL number at 0.5 in the simulations. The maximum CFL number is defined as

$$
C F L_{\max }=\max \left(u_{f i} d t\left(\frac{1}{d_{1}}+\frac{1}{d_{2}}\right)\right), \quad i=1,2,3, \ldots, \text { number of face, }
$$

where $u_{f i}$ is the normal component of the velocity at face $i$, and $d_{l}$ and $d_{2}$ the distances between the centroid of face $i$ and the centroids of its two neighboring cells, respectively. The uniform flow ( $U, 0$, 0 ) is specified at the inlet, the free convection boundary condition is used at the outlet. The non-slip boundary condition is set at the surface of the plate. The symmetric boundary conditions are used at the other boundaries. The flow is uniform $(U, 0,0)$ at $t=0$ before the plate instantaneously appears at $t=0^{+}$.

To validate the present numerical method by comparing with the published results by Taira and Colonius ${ }^{34}$ flows around an impulsively starting rectangular flat-plate wing with the aspect ratio $A R$ $=2$ are simulated at different angles of attack (AoA, $\alpha$ ) for the Reynolds number based on the chord $R e_{c}=100$. The lift and drag coefficients of the flat-plate wing calculated as a function of AoA are in good agreement with the numerical and experimental results obtained by Taira and Colonius. ${ }^{34}$ More details about the numerical method and its validations are given in our previous work. ${ }^{33}$ In Appendix B, three additional examples of flows over stationary and moving flat plate are given to compare our computational results with the existing experimental and numerical data.

\section{FLAT PLATE}

\section{A. Stationary plate}

The sectional lift of a stationary flat plate (a flat-plate airfoil) is calculated by using the lift formula $L^{\prime}=\rho U_{e f f} \Gamma-\rho D\left\langle\partial u_{z} / \partial t\right\rangle_{D}+\rho V_{B}\langle q \partial q / \partial z\rangle$ in comparison with the K-J theorem $L_{K-J}^{\prime}$ $=\rho U_{\infty} \Gamma$. The domain $D$ for the circulation integral and the lift formula is a sufficiently large area on the cross-section plane $(x, z)$. Figure 2 shows typical rectangular domains used here. The left boundary is located at $x / c=-1$ upstream the leading edge, the right boundary at the trailing edge and two locations in the wake, and the top and bottom boundaries at $z / c= \pm 6$, where $c$ is the chord. It should be pointed that there is no theory to determine the domain used in calculating the circulation 


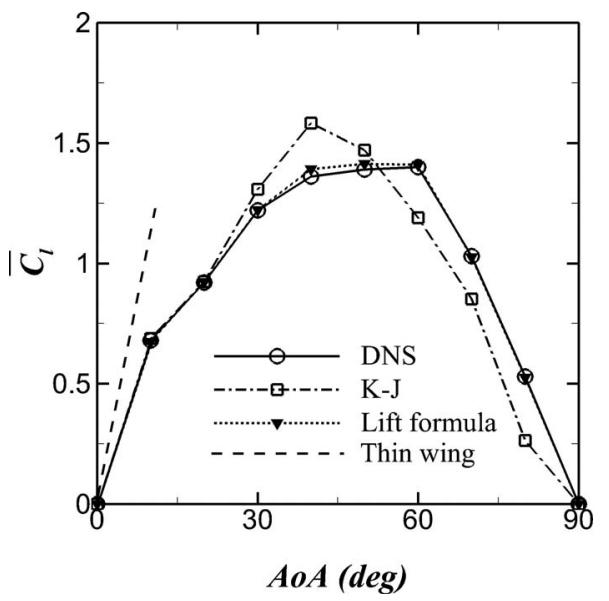

FIG. 3. The time-averaged lift coefficients of the flat plate as a function of AoA for $R e_{c}=300$, where "DNS" denotes the result calculated directly from the surface pressure and skin friction fields, "K-J" denotes the K-J theorem, "Lift formula" denotes the lift formula [Eq. (3) or (4)], and "Thin wing" denotes the thin-wing theory.

around the wing when the flow is fully separated and unsteady. The application of the K-J theorem depends on the selection of the domain, which reflects a problem in applying the K-J theorem to such flows. This problem is not addressed here. The domain used in calculating the circulation for the K-J theorem for comparison is the same as that for the lift formula (e.g., the domain D1).

For a stationary flat plate in the incoming freestream flow that starts suddenly, as shown in Figure 3, the time-averaged lift coefficients calculated by using the K-J theorem and the lift formula as a function of AoA are directly compared with those obtained from Eq. (1) for $R e_{c}=300$. The time-averaged lift coefficient is defined as $\overline{C_{l}}=\overline{L^{\prime}} /\left(\rho U_{\infty}^{2} c / 2\right)$, where the bar - denotes the timeaverage. In the relevant figures in this paper, "DNS" denotes the result calculated directly from Eq. (1) based on the surface pressure and stress distributions, "K-J" denotes the K-J theorem, "Lift formula" denotes the lift formula [Eq. (3) or (4)], and "Thin wing" denotes the thin-wing theory. The K-J theorem predicts the time-averaged lift coefficients $\overline{C_{l}}$ well for $\alpha \leq 30^{\circ}$, but it over-predicts $\overline{C_{l}}$ in $30^{\circ}<\alpha \leq 50^{\circ}$ and considerably under-predicts $\overline{C_{l}}$ for $\alpha>50^{\circ}$. The prediction by the classical thin-wing theory $\overline{C_{l}}=2 \pi \alpha$ is also plotted in Figure 3 as a reference. In contrast, the lift formula gives $\overline{C_{l}}$ that is in excellent agreement with DNS. Table I lists the time-averaged lift and drag coefficients of the flat plate at different AoAs along with the vortex shedding Strouhal numbers. The error in $\overline{C_{l}}$

TABLE I. Vortex shedding Strouhal number, time-averaged lift, and drag coefficients of the stationary 2D flat plate.

\begin{tabular}{lllllcrr}
\hline \hline$\alpha$ & $S t$ & $\bar{C}_{d}{ }^{\mathrm{a}}$ & $\bar{C}_{l}$ & $\bar{C}_{l, L F}{ }^{\mathrm{b}}$ & Error of $\bar{C}_{l, L F}$ & $\bar{C}_{l, K-J}$ & Error of $\bar{C}_{l, K-J}$ \\
\hline 0 & $\ldots$ & 0.2 & 0.0 & 0.0 & $\ldots$ & 0.0 & $\ldots$ \\
10 & $\ldots$ & 0.25 & 0.68 & 0.67 & $1 \%$ & 0.69 & $1 \%$ \\
20 & 0.16 & 0.45 & 0.92 & 0.92 & 0 & 0.92 & 0 \\
30 & 0.17 & 0.83 & 1.22 & 1.22 & 0 & 1.31 & $7 \%$ \\
40 & 0.17 & 1.30 & 1.36 & 1.39 & $2 \%$ & 1.58 & $16 \%$ \\
50 & 0.15 & 1.88 & 1.39 & 1.41 & $1 \%$ & 1.47 & $15 \%$ \\
60 & 0.15 & 2.62 & 1.40 & 1.41 & $1 \%$ & 1.19 & $17 \%$ \\
70 & 0.14 & 3.02 & 1.03 & 1.02 & $1 \%$ & 0.85 & $50 \%$ \\
80 & 0.14 & 3.23 & 0.53 & 0.52 & $2 \%$ & 0.26 & $\ldots$ \\
90 & 0.14 & 3.34 & 0.0 & 0.0 & $\ldots$ & 0.0 & $\ldots$ \\
\hline \hline
\end{tabular}

${ }^{\mathrm{a}} \bar{C}_{d}$ and $\bar{C}_{l}$ denote the time-averaged lift and drag coefficients directly obtained from DNS.

${ }^{\mathrm{b}} \bar{C}_{l, L F}$ and $\bar{C}_{l, K-J}$ denote the time-averaged lift coefficients given by using the simplified lift formula and the K-J theorem, respectively. 


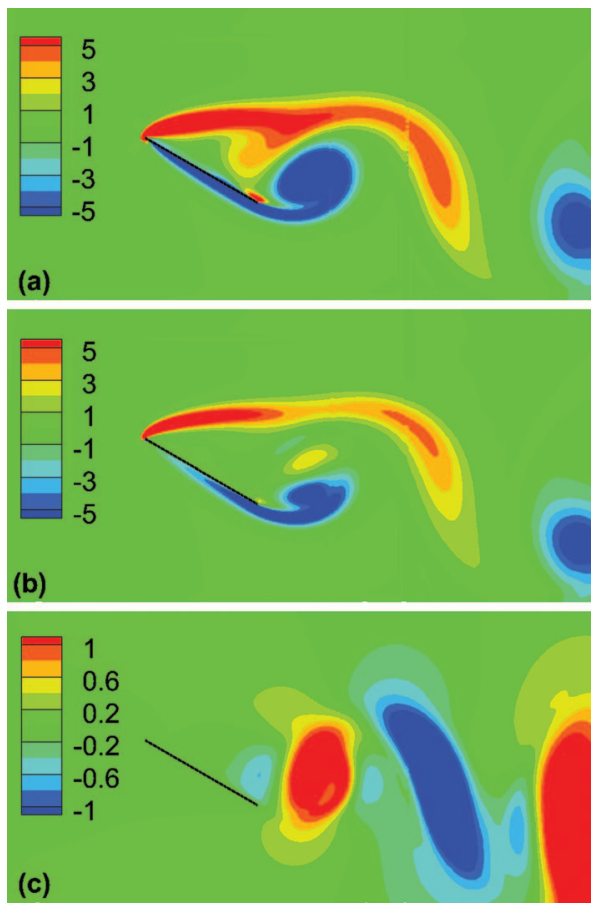

FIG. 4. Non-dimensional snap-shot fields around the stationary flat plate for $R e_{c}=300$ and $\alpha=30^{\circ}$, (a) vorticity, (b) vertically projected Lamb vector, and (c) vertically projected acceleration.

given by the K-J theorem is less than $7 \%$ for $\alpha \leq 30^{\circ}$, but it increases to about $20 \%$ in $30^{\circ}<\alpha \leq$ $50^{\circ}$. The error in $\overline{C_{l}}$ given by the lift formula is less than $2 \%$ for $10^{\circ}<\alpha \leq 80^{\circ}$.

Figure 4 shows the snap-shot fields of the non-dimensional vorticity, vertically projected Lamb vector, and vertically projected acceleration around a flat plate at the non-dimensional time $t^{*}=$ 147.6 for $R e_{c}=300$ and $\alpha=30^{\circ}$. Throughout this paper, the non-dimensional vorticity, vertically projected Lamb vector, vertically projected acceleration and time are defined as $\omega^{*}=\omega\left(c / U_{\infty}\right), \boldsymbol{k}$. $(\boldsymbol{u} \times \boldsymbol{\omega})^{*}=\boldsymbol{k} \cdot(\boldsymbol{u} \times \boldsymbol{\omega})\left(c / U_{\infty}^{2}\right), \boldsymbol{k} \cdot(\partial \boldsymbol{u} / \partial t)^{*}=\boldsymbol{k} \cdot \partial \boldsymbol{u} / \partial t\left(c / U_{\infty}^{2}\right)$, and $t^{*}=t\left(U_{\infty} / c\right)$, respectively. According to Eq. (3), the vertically projected Lamb vector and vertically projected acceleration are the leading terms contributing to the lift. Vortex shedding occurs when $\alpha \geq 20^{\circ}$ even though the flat plate is stationary, and therefore $C_{l}$ is time dependent. The vortex-shedding Strouhal number based on the front-projected height, defined as $S t=f_{s} c \sin \alpha / U_{\infty}$, is $S t=0.14-0.17$ as shown in Table I, which is consistent with the experimental data for flat-plates. ${ }^{35}$ Figure 5(a) shows the time histories
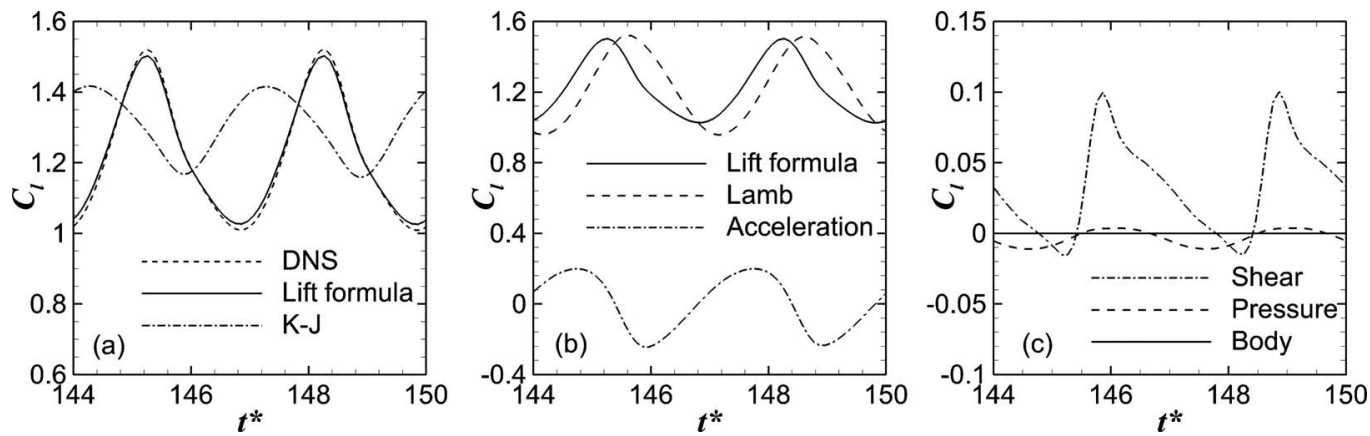

FIG. 5. Time histories of the lift coefficient of the stationary flat plate for $R e_{c}=300$ and $\alpha=30^{\circ}$, (a) comparison between DNS, the K-J theorem, and the lift formula, and (b) contributions from the Lamb vector integral and acceleration term, and (c) contributions from the shear stress, and total pressure term and the body boundary term. 
of the lift coefficient of the stationary plate for $R e_{c}=300$ and $\alpha=30^{\circ}$. The lift formula gives $C_{l}$ that is in good agreement with DNS in both the amplitude and phase. In this case, as shown in Figure 5(b), the Lamb vector integral is the major contribution to the lift, while the acceleration term has a relatively small effect. As indicated in Figure 4(c), the vertically projected acceleration around the flat plate is indeed small. Figure 5(c) shows the contributions from the total pressure, shear stress, and body boundary terms [the third, fourth, and fifth terms in RHS of Eq. (2), respectively] to the lift coefficient. The time-averaged contributions of the total pressure, shear stress, and body boundary terms to the lift coefficient are $0.03,0.003$, and 0 , respectively. The relative errors in the time-averaged lift due to neglecting the total pressure and shear stress terms are about $2 \%$ and $0.2 \%$, respectively. Clearly, their contributions to the lift generation can be neglected, which is consistent with the analysis in Sec. II. Note that the contribution of the body boundary term (the part of the added mass force associated with the fluid virtually occupying the body domain) is zero since the flat plate has zero volume theoretically.

Even for the stationary plate, as indicated in Figure 5(a), there is a large phase difference between $L^{\prime}(t)$ given by the lift formula and $L_{K-J}^{\prime}(t)$ by the K-J theorem. The amplitude given by the K-J theorem is lower than that given by the lift formula particularly when the flow becomes unsteady for large AoAs. The K-J theorem $L_{K-J}^{\prime}(t)=\rho U_{\infty} \Gamma(t)$ indicates that the lift on the flat plate has the same phase with the circulation around the wing since $U_{\infty}$ is a constant for a stationary flat plate. However, the lift formula $L^{\prime}=\rho U_{e f f} \Gamma-\rho D\left\langle\partial u_{z} / \partial t\right\rangle_{D}+\rho V_{B}\langle q \partial q / \partial z\rangle$ shows that the lift is out of phase with the circulation due to not only the time-dependent effective velocity $U_{\text {eff }}(t)$ but also the unsteady acceleration term $\left\langle\partial u_{z} / \partial t\right\rangle_{D}$. In this case, $L^{\prime}(t)$ is out of phase with $\Gamma(t)$ by about $180^{\circ}$ and the phase of $L^{\prime}(t)$ is dominated by the phase of $U_{\text {eff }}(t)$. The K-J theorem as a quasi-steady model does not reflect the unsteady coupling between $U_{\text {eff }}(t)$ and $\Gamma(t)$. The time-averaged lift difference given by the generalized lift formula and the K-J theorem is $\overline{L^{\prime}}-\bar{L}_{K-J}=\rho C_{v \Gamma}|\bar{v}||\bar{\Gamma}|-\rho D\left\langle\overline{\partial u_{z} / \partial t}\right\rangle_{D}+\rho V_{B}\langle\overline{q \partial q / \partial z}\rangle_{B}$, where a correlation coefficient $C_{v \Gamma}=\overline{v \Gamma} /|\bar{v}||\bar{\Gamma}|$ is introduced. This difference is proportional to $\bar{v}=U_{\infty}\left[\overline{U_{e f f}} / U_{\infty}-1\right]$. It is found that $\overline{U_{e f f}} / U_{\infty}$ increasingly deviates from one as AoA increases after $\alpha>30^{\circ}$, which corresponds to the difference $\overline{L^{\prime}}-{\overline{L^{\prime}}}_{K-J}$ shown in Figure 3.

In the derivation of the lift formula Eq. (3), the rectangular domain is not specified. Theoretically speaking, lift calculation is independent of a rectangular domain selected, but actual result is affected by the selection of a domain depending on the numerical or measurement accuracy. To illustrate this issue, three rectangular domains shown in Figure 2 are used for Eq. (3). Figure 6 shows the time histories of the lift coefficient of the stationary flat plate calculated based on the three domains for $R e_{c}=300$ and $\alpha=30^{\circ}$. The value of $C_{l}$ calculated based on the domain $D_{1}$ where the right boundary is at the trailing edge is very close to DNS. In contrast, calculations based on the domains $D_{2}$ and $D_{3}$ that contain the portion of the wake structures under-predict $C_{l}$ somewhat particularly

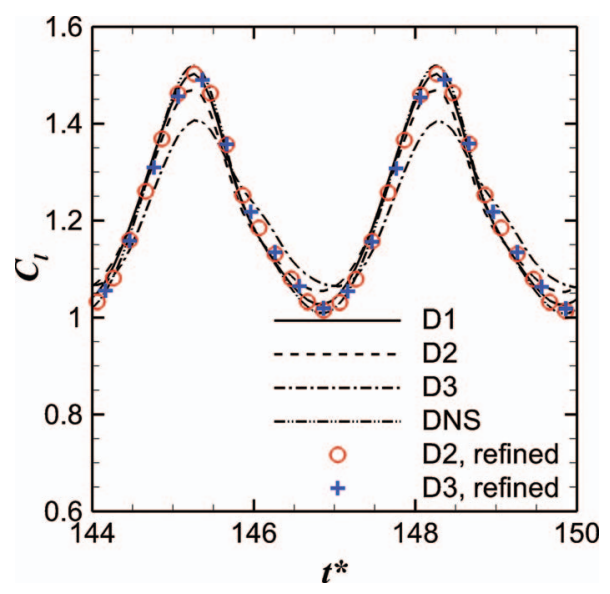

FIG. 6. Time histories of the lift coefficient of the stationary plate calculated based on three domains for $R e_{c}=300$ and $\alpha=30^{\circ}$. 


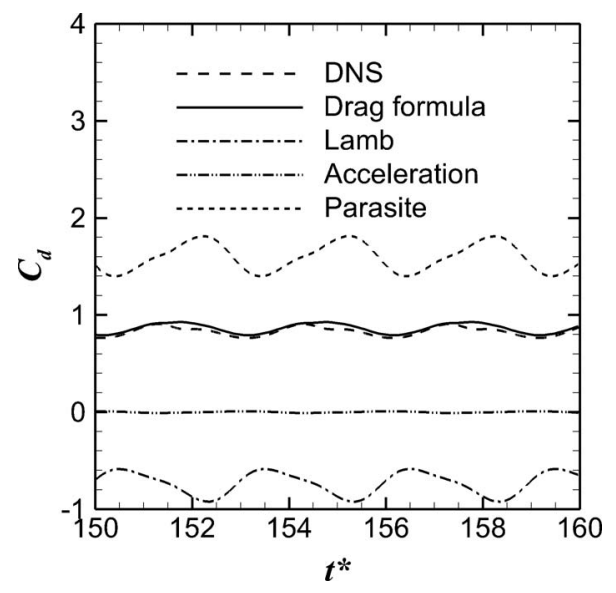

FIG. 7. Time histories of the drag coefficient of the stationary flat plate for $R e_{c}=300$ and $\alpha=30^{\circ}$ for comparison between DNS and the drag formula, where the contributions from the Lamb vector integral, acceleration term, and parasite drag are also included.

near the peaks. The more pronounced deviation is found for the larger domain $D_{3}$. This larger error found for a larger domain is not directly related to the size of the domain and the accuracy of the force formula itself. This is caused by the numerical errors in a coarser grid in the wake region in the larger domain, which leads to underestimation of the vorticity and lift as a result. This error decreases when the grid in the wake region is refined, as shown in Figure 6. The main results in this paper are obtained based on the domain $D_{1}$.

Figure 7 shows the time histories of the drag coefficient of the stationary flat plate for $R e_{c}=$ 300 and $\alpha=30^{\circ}$ for comparison between DNS and the drag formula, where the contributions from the Lamb vector integral, acceleration term and parasite drag are also included. The drag coefficient calculated by using Eq. (8) is compared well with the DNS result. The time-averaged drag coefficient given by Eq. (8) is 0.86 compared to 0.83 from DNS, and the relative error is about $4 \%$. The parasite drag associated with the total pressure term is dominant while the contribution from the acceleration is negligible. The Lamb vector integral contributes a thrust in this case.

\section{B. Flapping plate}

The applicability of the K-J theorem and the lift formula to a flapping plate is examined. The kinematics of a flapping flat plate is prescribed as a superposition of the pitching and heaving motions by

$$
\alpha=\alpha_{0}+\alpha_{m} \cos (2 \pi f t), \quad z_{c}=A \sin (2 \pi f t),
$$

where the time-averaged AoA is $\alpha_{0}=10^{\circ}$, the pitching amplitude is $\alpha_{m}=30^{\circ}$, the heaving amplitude is $A=c / 4, z_{c}$ is the vertical position of the plate center, and $f$ is the flapping frequency. The flapping Strouhal number is $S t_{f}=2 A f / U_{\infty}=0.3$ that is close the optimal Strouhal number $\left(0.2<S t_{f}<0.4\right)$ of various flying and swimming animals for high power efficiency. ${ }^{36}$ The corresponding reduced frequency is $f c / U_{\infty}=0.6$ The total sectional force on the plate calculated by using the K-J theorem is $F^{\prime}=\rho U \Gamma$, where $U=\sqrt{U_{\infty}^{2}+U_{c}^{2}}$ is the effective local upstream velocity, $U_{\infty}$ is the freestream velocity, $U_{c}=\dot{z}_{c}=2 \pi f A \cos (2 \pi f t)$ is the heaving velocity of the plate center. The direction of the effective upstream velocity relative to the rotating center at the plate middle span is given by $\theta=-\arctan \left(-U_{c} / U_{\infty}\right)$. The sectional lift is the vertical component of the total force $L^{\prime}=F^{\prime} \cos (\theta)$.

Figure 8 shows the snap-shot fields of the non-dimensional vorticity, vertically projected Lamb vector, and vertically projected acceleration around the flapping flat plate at $t^{*}=18.8$ when $C_{l}$ reaches a peak for $R e_{c}=300$. Compared with Figure 4 for the stationary plate, the significant vertically projected acceleration of fluid is found around the flapping plate, which contributes to the unsteady lift. Figure 9(a) shows the histories of the lift coefficient of the flapping flat plate. The lift 


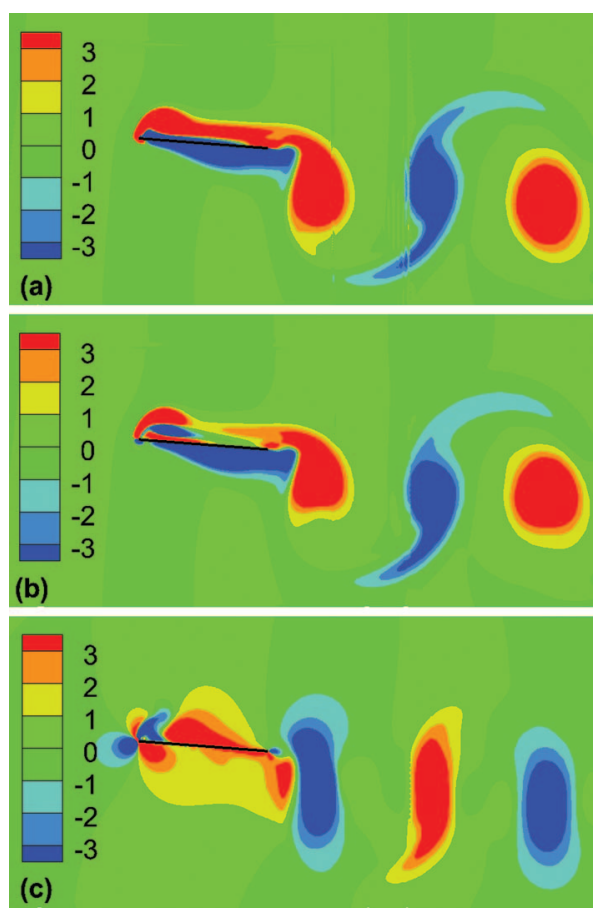

FIG. 8. Non-dimensional snap-shot fields around the flapping flat plate at $t^{*}=18.8$ when $C_{l}$ reaches a peak for $R e_{c}=300$, (a) vorticity, (b) vertically projected Lamb vector, and (c) vertically projected acceleration.

formula gives the result that is consistent with DNS. The contributions from the Lamb vector integral and acceleration term are shown in Figure 9(b). The Lamb vector integral mainly contributes to the mean lift with a considerable phase shift compared to DNS, while the acceleration term significantly modifies the waveform and phase. The sum of the Lamb vector integral and the acceleration term recovers the true waveform. As shown in Figure 9(d), the contributions from the total pressure, shear stress, and body boundary terms in (2) to the lift generation are small. The time-averaged lift coefficient components of the total pressure, shear stress, and body boundary terms are -0.03 , 0.001 , and 0 , respectively. The relative errors in the time-averaged lift due to neglecting the total pressure and shear stress terms are about $1.5 \%$ and $0.1 \%$, respectively.

Theodorsen ${ }^{37}$ gave an analytical expression of the lift of a harmonically flapping flat plate based on potential flow theory, which has the non-circulatory and circulatory terms. The non-circulatory term is essentially the added mass force generated purely by the unsteady motion of the plate. The circulatory term is the quasi-steady lift modulated by Theodorsen's function depending on the reduced frequency to take the effect of the infinite straight wake into account. In Figure 9(a), Theodorsen's formula is compared with DNS, the K-J theorem, the lift formula, which considerably under-predicts both the amplitude and the time-averaged value of the lift. Theodorsen's formula gives the time-averaged lift coefficient of 0.56 that is much smaller than 2.07 given by DNS and the maximum lift that is about one half of the value given by DNS. Physically speaking, the noncirculatory and circulatory terms in Theodorsen's formula correspond to the acceleration term and the Lamb vector integral in the lift formula, respectively. Figure 9(c) shows comparisons between these terms in this case. It is found that the Theodorsen's non-circulatory and circulatory terms are in phase with the acceleration term and the Lamb vector integral, respectively. Therefore, Theodorsen's formula correctly predicts the phase of the lift unlike the K-J theorem that is a quasi-steady model. However, the amplitude of the non-circulatory term as the added mass force is considerably smaller than the corresponding acceleration term in Eq. (3). The non-circulatory term does not contribute to the time-averaged lift because of the symmetric flapping kinematics in downstroke and upstroke. The time-averaged value of the circulatory term in Theodorsen's formula is also much smaller than that of the Lamb vector integral. The sinusoidal waveform of the lift calculated by Theodorsen's 

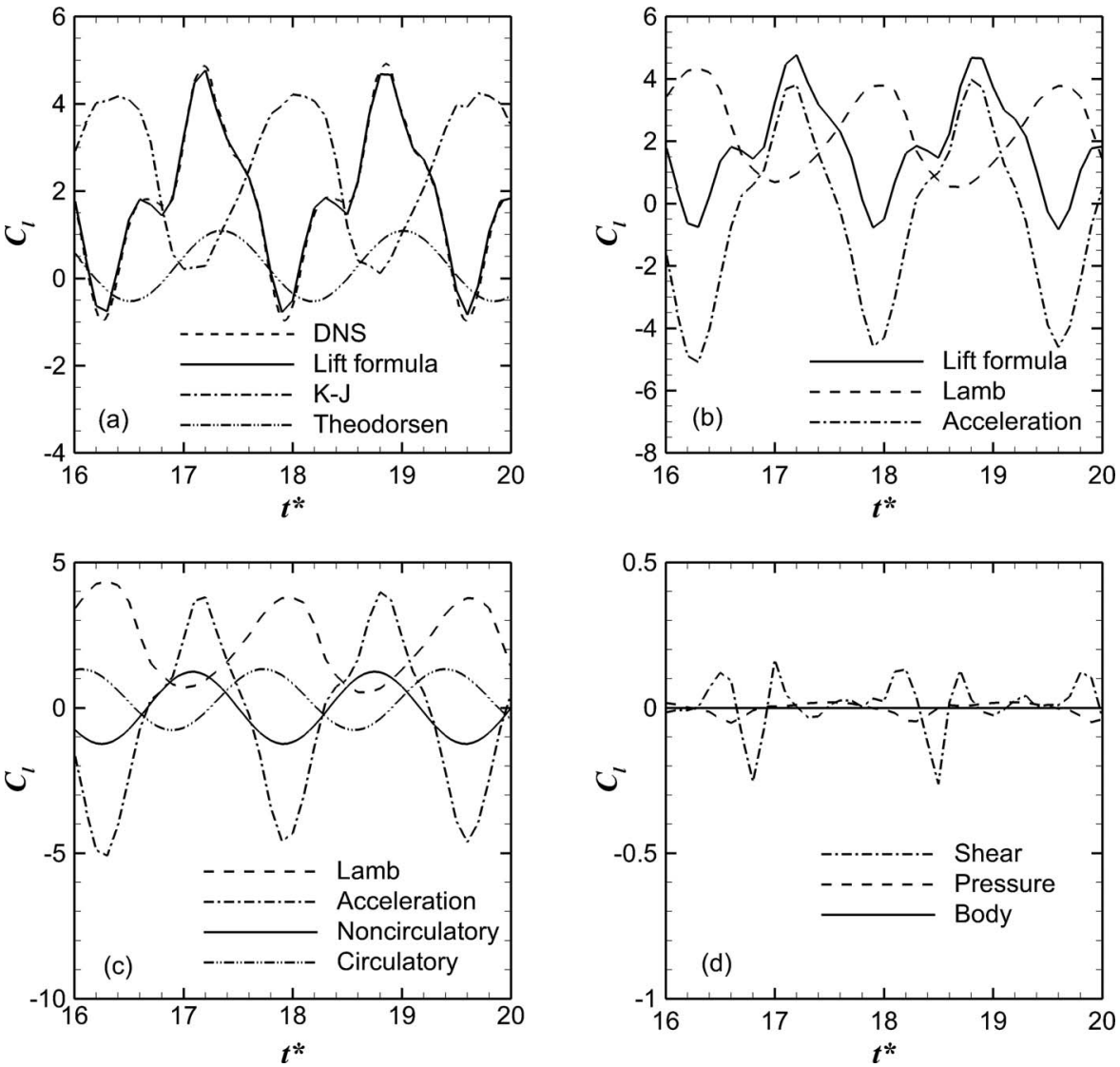

FIG. 9. Histories of the lift coefficient of the flapping flat plate for $R e_{c}=300$, (a) comparison between DNS, the K-J theorem, Theodorsen's formula, and the lift formula, and (b) contributions from the Lamb vector integral and acceleration term, (c) comparison with the non-circulatory and circulatory terms in Theodorsen's formula, and (d) contributions from the shear stress, and total pressure term and the body boundary term.

formula is different from the more complex waveform given by DNS. These differences are not unexpected since Theodorsen's model as a linear potential flow theory is not able to describe some major unsteady separated flow features particularly vortical structures generated from the leading and trailing edges of the plate. Therefore, Theodorsen's model does not take into account the nonlinear effects associated with these structures. For a pitching and plunging airfoil with a round leading edge where separation is moderate at higher Reynolds numbers $\left(\sim 10^{4}\right)$ when the pitching and plunging amplitudes are small, Theodorsen's model is able to give a reasonable prediction..$^{38,39}$

The time-averaged lift coefficients calculated based on different methods are showed in Table II along with the contributions of the Lamb vector integral and acceleration term to the time-averaged lift. It is shown that the time-averaged lift calculated by using the K-J theorem just captures the contribution of Lamb vector integral. In this case, the flapping kinematics of the plate is

TABLE II. Time-averaged lift coefficient of the flapping flat plate.

\begin{tabular}{lcccc}
\hline \hline $\bar{C}_{l}^{\mathrm{a}}$ & $\bar{C}_{l, L F}{ }^{\mathrm{b}}$ & $\bar{C}_{l, K-J}$ & $\bar{C}_{l, \text { Lamb }} \mathrm{c}^{\mathrm{c}}$ & $\bar{C}_{l, a c c}$ \\
\hline 2.07 & 2.07 & 1.86 & 1.86 & 0.21 \\
\hline \hline
\end{tabular}

${ }^{\mathrm{a}} \bar{C}_{d}$ and $\bar{C}_{l}$ denote the time-averaged lift and drag coefficients directly obtained from DNS.

${ }^{\mathrm{b}} \bar{C}_{l, L F}$ and $\bar{C}_{l, K-J}$ denote the time-averaged lift coefficients given by using the simplified lift formula and the K-J theorem, respectively.

${ }^{\mathrm{c}} \bar{C}_{l, \text { Lamb }}$ and $\bar{C}_{l, a c c}$ represent the contributions of the Lamb vector integral and acceleration term in the general lift formula, respectively. 


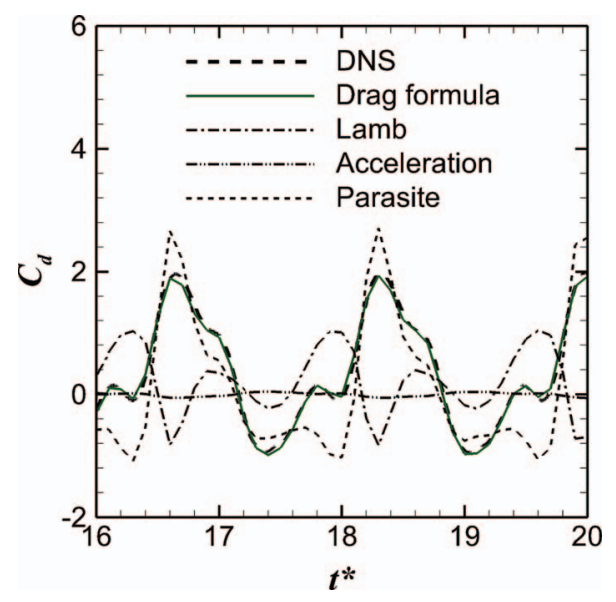

FIG. 10. Time histories of the drag coefficient of the flapping flat plate for $R e_{c}=300$ for comparison between DNS and the drag formula, where the contributions from the Lamb vector integral, acceleration term, and parasite drag are also included.

not symmetric with respect to the time axis, the time-averaged quantity $\left\langle\overline{\partial u_{z} / \partial t}\right\rangle_{D}$ contributes about $10 \%$ of the time-averaged lift. The more asymmetric flapping of a bird or bat may contribute more to the mean lift through the acceleration term. When the time-averaged AoA in Eq. (14) is $\alpha_{0}=$ $0^{\circ}$ in the symmetric flapping kinematics, the time-averaged contribution of the acceleration term is zero. The above analysis indicates that a quasi-steady model like the K-J theorem (even the Lamb vector integral alone) based on the snap-shot vorticity fields cannot capture the unsteady nature of the lift in flapping flight. The acceleration term for the unsteady inertial effect is significant, and time-resolved velocity measurements or computations are required to obtain the unsteady lift.

Figure 10 shows the time histories of the drag coefficient of the flapping flat plate for $R e_{c}=300$ for comparison between DNS and the drag formula, where the contributions from the Lamb vector integral, acceleration term and parasite drag are also included. The parasite drag is still dominant while the contribution from the acceleration is negligible. In this case, the Lamb vector integral does not generate a thrust in a time-averaging sense. The time-averaged drag coefficient calculated by using Eq. (8) is 0.31, while DNS gives 0.34. The relative error of the drag calculation is $9 \%$.

\section{RECTANGULAR FLAT-PLATE WING}

\section{A. Stationary wing}

Flow fields over a stationary rectangular flat-plate wing with $A R=4$ are calculated at different AoAs for $R e_{c}=300$ after the incoming freestream flow starts suddenly. For a finite wing, Eq. (3) can be expressed as

$$
L=\rho V_{D}\left(\left\langle u_{x} \omega_{y}\right\rangle_{D}-\left\langle u_{y} \omega_{x}\right\rangle_{D}\right)-\rho V_{D}\left\langle\partial u_{z} / \partial t\right\rangle_{D}+\rho V_{B}\langle q \partial q / \partial z\rangle_{B} .
$$

The first term in the Lamb vector integral on RHS of Eq. (15) is the dominant component, and the second term is a relatively small component for a rectangular wing since interaction between the spanwise velocity and the streamwise vorticity is weak. Figure 11 shows the time-averaged lift coefficient as a function of AoA. The lift formula predicts well the lift for all AoAs. In planar PIV measurements, 2D velocity fields on different spanwise slices along the wing span are usually obtained, and therefore the sectional lift $L^{\prime}$ is calculated for each slice. Then, the total lift $L$ of the wing is obtained by summing the contributions from all the slices. This approach is a quasi-2D approximation where only the first term in the Lamb vector integral in Eq. (15) is used since the streamwise vorticity and the spanwise velocity cannot be measured at the same time in planar PIV. The lift formula is compared with DNS in Figure 11. It is indicated that this quasi-2D approximation is reasonably good even though the time-averaged lift is over-predicted slightly for $\alpha>30^{\circ}$. Interestingly, in this case, the K-J theorem gives the time-averaged lift that is consistent with DNS 

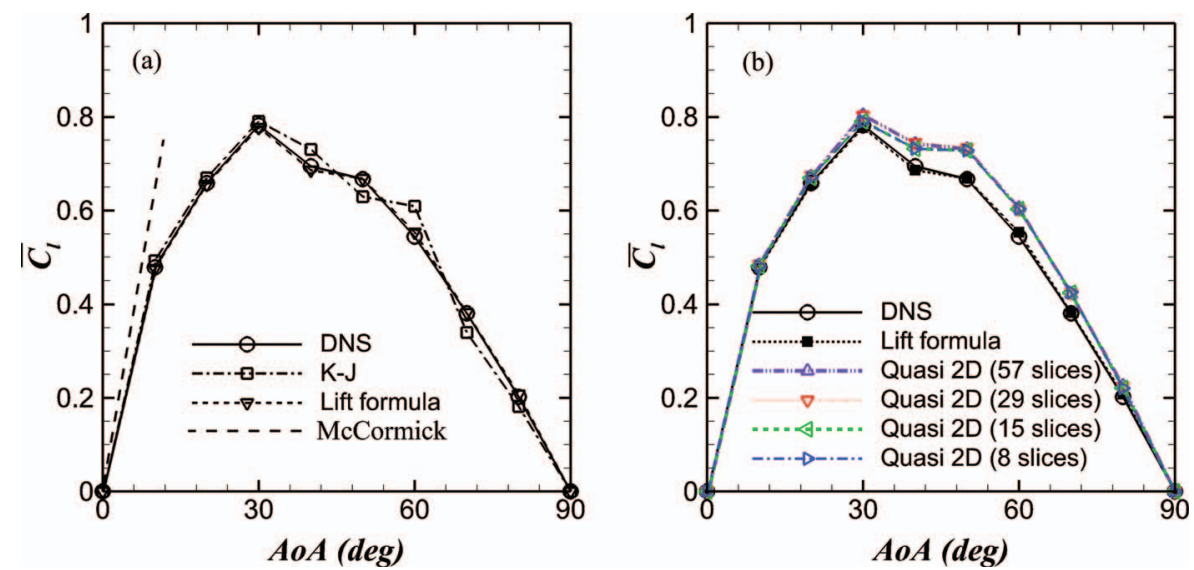

FIG. 11. The time-averaged lift coefficient as a function of AoA for the stationary rectangular flat-plate wing with $A R=4$ for $R e_{c}=300$, (a) comparison between DNS, the K-J theorem, and the lift formula and (b) quasi-2D estimation of the lift.

as well. This may be coincident. For comparison, Figure 11(a) also includes the lift for a finite wing given by McCormick's formula where the lift slope is ${ }^{40}$

$$
a=\frac{d C_{l}}{d \alpha}=\frac{a_{0} A R}{A R+2(A R+4) /(A R+2)},
$$

where $a_{0}=2 \pi$ according to the thin-wing theory.

Figure 12 shows the non-dimensional snap-shot iso-surface fields of the vorticity, vertically projected Lamb vector, and vertically projected acceleration around a stationary rectangular

(a)

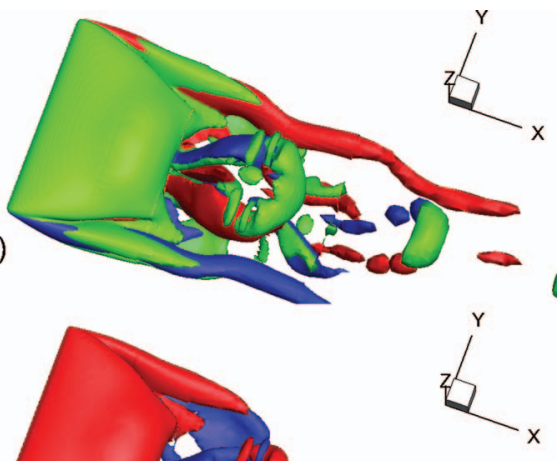

(b)

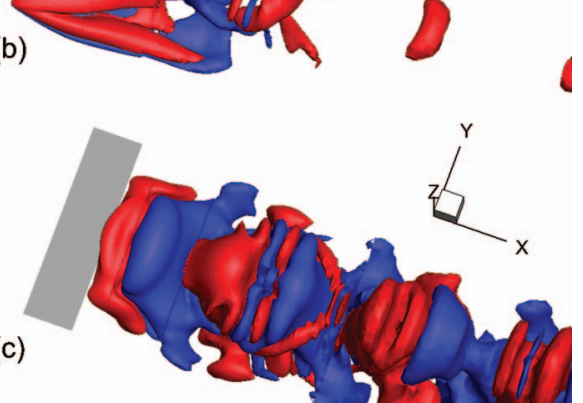

FIG. 12. Non-dimensional snap-shot iso-surface fields around the stationary rectangular flat-plate wing with $A R=4$ at $t^{*}$ $=98.9$ when $-\left\langle\partial u_{z} / \partial t\right\rangle_{D}$ reaches the maximum for $R e_{c}=300$ and $\alpha=30^{\circ}$, (a) iso-surfaces of vorticity, the lightest gray (or green): $\omega_{y}^{*}= \pm 1.0$, the darkest gray (or blue): $\omega_{x}^{*}=-1.0$, mid-gray (or red): $\omega_{x}^{*}=1.0$, (b) iso-surfaces of vertically projected Lamb vector, the darkest gray (or blue): $\boldsymbol{k} \cdot(\boldsymbol{u} \times \boldsymbol{\omega})^{*}=-1.0$, mid-gray (or red): $\boldsymbol{k} \cdot(\boldsymbol{u} \times \boldsymbol{\omega})^{*}=1.0$, and (c) isosurfaces of vertically projected acceleration, the darkest gray (or blue): $\left(-\partial u_{z} / \partial t\right)^{*}=-0.25$, mid-gray (or red): $\left(-\partial u_{z} / \partial t\right)^{*}$ $=0.25$ ). 

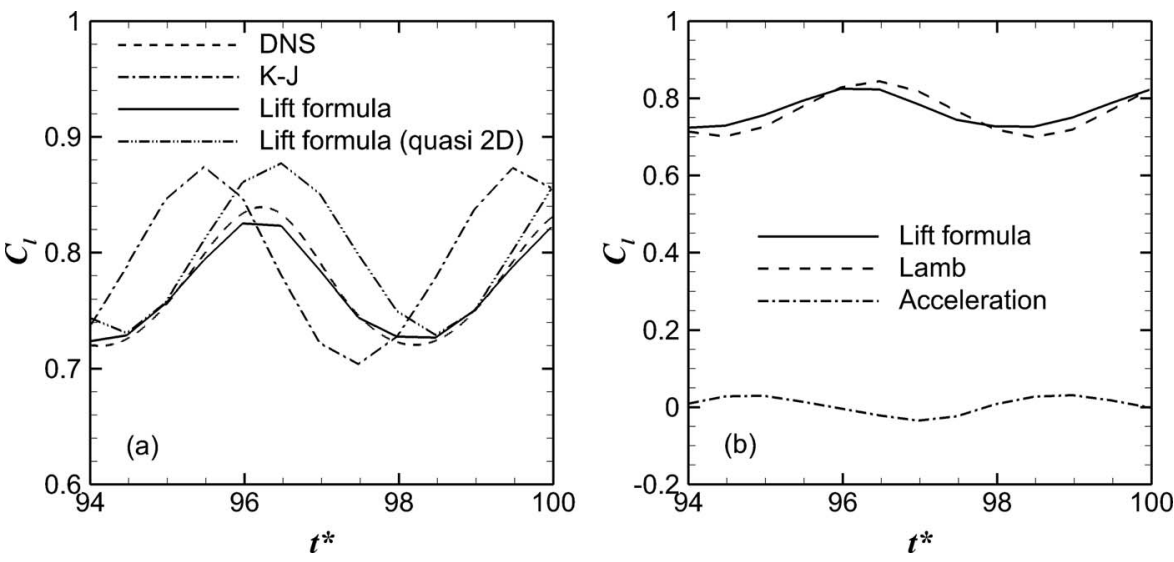

FIG. 13. Histories of the lift coefficient of the stationary rectangular flat-plate wing with $A R=4$ for $R e_{c}=300$ and $\alpha=30^{\circ}$, (a) comparison between DNS, the K-J theorem, the lift formula, and quasi-2D approximation and (b) contributions from the Lamb vector integral and acceleration term.

flat-plate wing with $A R=4$ at $t^{*}=98.9$ when $\left\langle\partial u_{z} / \partial t\right\rangle_{D}$ reaches the maximum for $R e_{c}=300$ and $\alpha=30^{\circ}$. For the vorticity iso-surfaces, the lightest gray (or green), the darkest gray (or blue), and mid-gray (or red) correspond to $\omega_{y}^{*}= \pm 1.0, \omega_{x}^{*}=-1.0$, and $\omega_{x}^{*}=1.0$, respectively. For the vertically projected Lamb vector iso-surfaces, the darkest gray (or blue) and mid-gray (or red) respond to $\boldsymbol{k} \cdot(\boldsymbol{u} \times \boldsymbol{\omega})^{*}=-1.0$ and $\boldsymbol{k} \cdot(\boldsymbol{u} \times \boldsymbol{\omega})^{*}=1.0$, respectively. For the vertically projected acceleration iso-surfaces, the darkest gray (or blue) and mid-gray (or red) correspond to $\left(-\partial u_{z} / \partial t\right)^{*}=-0.25$ and $\left(-\partial u_{z} / \partial t\right)^{*}=0.25$, respectively. The superscript “*” denotes non-dimensional quantities. Similar to Figure 4 for the 2D case, the vertically projected acceleration of fluid around the wing is small, indicating its contribution to the time-averaged lift is not significant. Figure 13(a) shows the histories of the lift coefficient of the wing for $R e_{c}=300$ and $\alpha=30^{\circ}$. The lift formula gives a consistent result with DNS. In contrast, the lift coefficient given by the K-J theorem has a lower time-averaged value and a significant phase shift. It is indicated that the first term the Lamb vector integral in Eq. (15) makes the most contribution to the lift from the spanwise vorticity field. Although the contribution of the acceleration to the time-averaged lift is relatively small for the stationary wing, it affects the phase as indicated in Figure 13(b).

\section{B. Flapping wing}

The kinematics of a flapping rectangular flat-plate wing is prescribed using Eq. (14). Figure 14 shows the non-dimensional snap-shot iso-surface fields around the flapping rectangular flat-plate wing with $A R=4$ at $t^{*}=23.7$ when $\left\langle\partial u_{z} / \partial t\right\rangle_{D}$ reaches the maximum for $\operatorname{Re}_{c}=300$. For the vorticity iso-surfaces, the lightest gray (or green), the darkest gray (or blue) and mid-gray (or red) correspond to $\omega_{y}^{*}= \pm 2.0, \omega_{x}^{*}=-2.0$ and $\omega_{x}^{*}=2.0$, respectively. For the vertically projected Lamb vector iso-surfaces, the darkest gray (or blue) and mid-gray (or red) respond to $\boldsymbol{k} \cdot(\boldsymbol{u} \times \boldsymbol{\omega})^{*}$ $=-2.0$ and $\boldsymbol{k} \cdot(\boldsymbol{u} \times \boldsymbol{\omega})^{*}=2.0$, respectively. For the vertically projected acceleration iso-surfaces, the darkest gray (or blue) and mid-gray (or red) correspond to $\left(-\partial u_{z} / \partial t\right)^{*}=-2.0$ and $\left(-\partial u_{z} / \partial t\right)^{*}$ $=2.0$, respectively. In contrast to the stationary wing shown in Figure 12 , the vertically projected acceleration around the flapping wing is significantly large. Figure 15(a) shows the histories of the lift coefficient of the flapping wing. The lift formula gives the consistent result with DNS. The lift predicted by the K-J theorem has not only a considerable phase shift but also a different waveform compared to DNS. Interestingly, in this case, the Lamb vector integral in the lift formula gives a waveform that is close to that given by the K-J theorem. Nevertheless, as indicated in Figure 15(b), the acceleration term for the unsteady inertial effect has a considerably large effect on both the amplitude and phase of the lift for the flapping wing. The sum of the Lamb vector integral and the acceleration term recovers the true waveform. Table III gives the time-averaged lift coefficients 
(a)
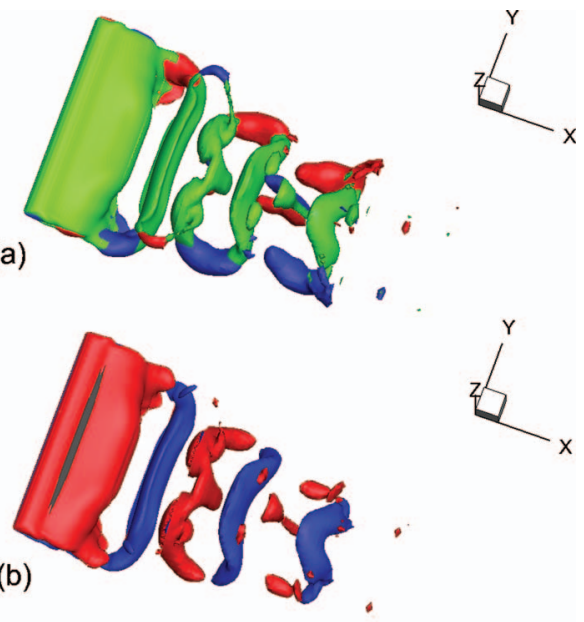

(c)

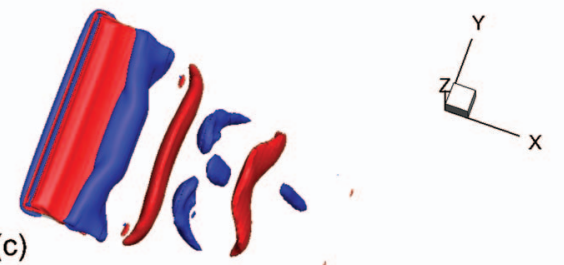

FIG. 14. Non-dimensional snap-shot iso-surface fields around the flapping rectangular flat-plate wing with $A R=4$ at $t^{*}$ $=23.7$ when $-\left\langle\partial u_{z} / \partial t\right\rangle_{D}$ reaches the maximum for $R_{c}=300$, (a) iso-surfaces of vorticity, the lightest gray (or green): $\omega_{y}^{*}= \pm 2.0$, the darkest gray (or blue): $\omega_{x}^{*}=-2.0$, mid-gray (or red): $\omega_{x}^{*}=2.0$, (b) iso-surfaces of vertically projected Lamb vector, the darkest gray (or blue): $\boldsymbol{k} \cdot(\boldsymbol{u} \times \boldsymbol{\omega})^{*}=-2.0$, mid-gray (or red): $\boldsymbol{k} \cdot(\boldsymbol{u} \times \boldsymbol{\omega})^{*}=2.0$, and (c) iso-surfaces of vertically projected acceleration, the darkest gray (or blue): $\left(-\partial u_{z} / \partial t\right)^{*}=-2.0$, mid-gray (or red): $\left(-\partial u_{z} / \partial t\right)^{*}=2.0$.

calculated based on different methods along with the contributions of the Lamb vector term and acceleration term. Similar to the 2D flow case in Table II, the K-J theorem does not capture the contribution of the flow acceleration to the time-averaged lift. The acceleration term $\left\langle\partial u_{z} / \partial t\right\rangle_{D}$ contributes about $10 \%$ of the time-averaged lift.
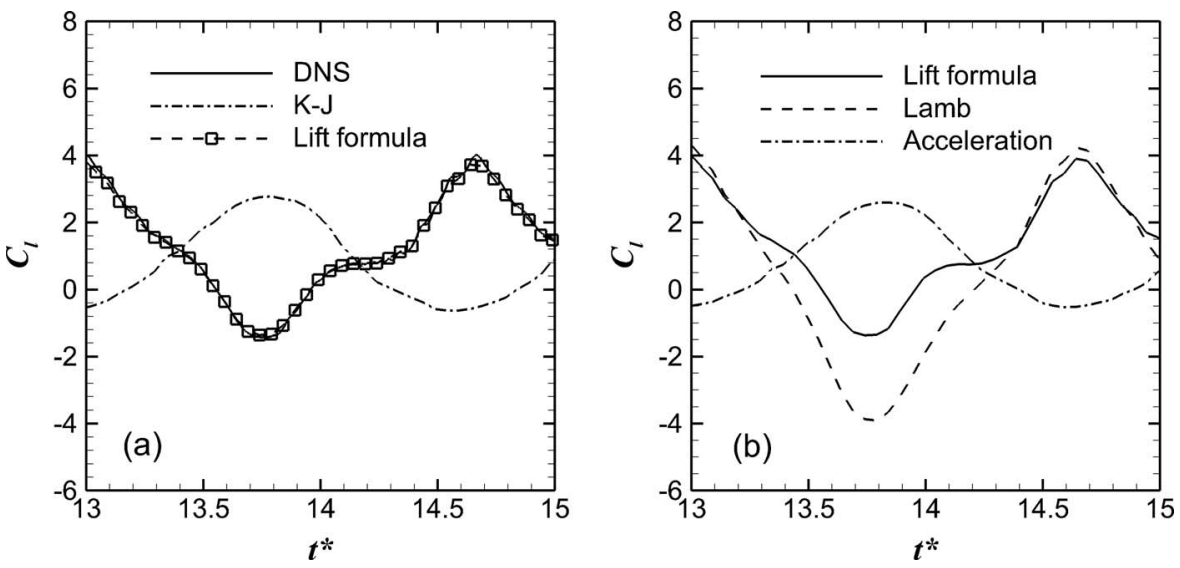

FIG. 15. Histories of the lift coefficient for the flapping rectangular flat-plate wing with $A R=4$ for $R e_{c}=300$, (a) comparison between DNS, the K-J theorem, and the lift formula and (b) contributions from the Lamb vector integral and acceleration term. 
TABLE III. Time-averaged lift coefficient of the flapping rectangular flat-plate wing.

\begin{tabular}{lcccr}
\hline \hline $\bar{C}_{l}^{\mathrm{a}}$ & $\bar{C}_{l, L F}{ }^{\mathrm{b}}$ & $\bar{C}_{l, K-J}$ & $\bar{C}_{l, \text { Lamb }}{ }^{\mathrm{c}}$ & $\bar{C}_{l, a c c}$ \\
\hline 1.13 & 1.12 & 1.01 & 1.02 & 0.10 \\
\hline
\end{tabular}

${ }^{\mathrm{a}} \bar{C}_{l}$ denote the time-averaged lift coefficients directly obtained from DNS.

${ }^{\mathrm{b}} \bar{C}_{l, L F}$ and $\bar{C}_{l, K-J}$ denote the time-averaged lift coefficients given by using the simplified lift formula and the K-J theorem, respectively.

${ }^{\mathrm{c}} \bar{C}_{l, \text { Lamb }}$ and $\bar{C}_{l, a c c}$ represent the contributions of the Lamb vector integral and acceleration term in the general lift formula, respectively.

\section{CONCLUSIONS}

The lift formula is given for a wing in a rectangular control volume in the framework of the general viscous flow theory, and it has a lucid form with the two leading-order terms: the Lamb vector integral for the vortex force and the acceleration term of fluid for the unsteady inertial effect. The acceleration term includes the added mass force and other unsteady effects associated with flow structures generated in complex flows. The lift formula is validated and the relevant theoretical arguments are supported by direct numerical simulation (DNS) on the stationary and flapping flat plate and rectangular flat-plate wing. As indicated by DNS, the fields of the vertically projected Lamb vector and vertically projected acceleration around a flapping wing are directly responsible to the lift generation. Due to its simplicity, the lift formula is useful for estimation and analysis of the unsteady lift from velocity fields obtained in measurements and computations. In addition, since the Kutta-Joukowski (K-J) theorem is a reduced case from this lift formula, the limitations of the K-J theorem can be critically examined. It is found that the application of the K-J theorem as a quasi-steady model to unsteady low-Reynolds-number flows may lead to errors in prediction of the phase, amplitude and waveform of the unsteady lift of a flapping wing.

\section{ACKNOWLEDGMENTS}

The authors would like to thank Professor Jiezhi Wu of Peking University and University of Tennessee Space Institute for his helpful discussions. This work was supported by the National Natural Science Foundation of China under Project Nos. 10872201, 11232011, 11302238, and 11372331 and the National Basic Research Program of China (973 Program) under Project No. 2013CB834100 (Nonlinear science). Tianshu Liu would like to acknowledge the hospitality received at LNM during his visit where he accomplished this work. The simulations were performed on TianHe-1A. The authors would like to acknowledge the support from National Supercomputer Center in Tianjin.

\section{APPENDIX A: PHYSICAL MEANINGS OF THE SECOND AND FIFTH TERMS OF RHS OF EQ. (2)}

The second term in RHS of Eq. (2), which is a volume integral of the local acceleration of fluid induced by a moving solid body, represents the unsteady inertial effect. The physical meanings of the second and fifth terms in RHS of Eq. (2) can be further illustrated in a reduced case when an inviscid irrotational flow is considered where the first, third, and fourth terms in RHS of Eq. (2) vanish. For an inviscid irrotational flow, when the outer control surface $\Sigma$ is time independent and the far-field flow is steady, Eq. (2) is reduced to

$$
\begin{aligned}
\boldsymbol{F} & =-\rho \int_{V_{f}} \frac{\partial \boldsymbol{u}}{\partial t} d V-\rho \oint_{\partial B}\left(q^{2} / 2\right) \boldsymbol{n} d S \\
& =-\rho \frac{d}{d t} \int_{V_{f}} \boldsymbol{u} d V+\rho \oint_{\Sigma} \boldsymbol{n} \cdot\left[\frac{1}{2} q^{2} \boldsymbol{I}-\boldsymbol{u} \boldsymbol{u}\right] d S .
\end{aligned}
$$


In fact, by some manipulations, it can be found that in a potential flow Eq. (A1) equals to the acceleration-reaction force given by Noca et al. ${ }^{20}$ Therefore, when the unsteadiness of an inviscid irrotational flow is exclusively associated with the motion of a moving body, the second and fifth terms together are reduced to the added mass force in ideal fluid mechanics.

To illustrate this point, a sphere moving with a velocity of $V_{s}(t)$ along the positive $z$-coordinate is considered as a classical case, and the velocity potential is $\phi=-V_{s} R^{3} \cos \theta /\left(2 r^{2}\right)$ in a spherical coordinate system and $\boldsymbol{u}=\nabla \phi$, where $R$ is the radius of the sphere, $\theta$ is the polar angle, and $r$ is the radial position. In the second equality in RHS of Eq. (A1), since $\phi \sim r^{-2}$ and $\nabla \phi \sim r^{-3}$ decay fast as $r \rightarrow \infty$, the volume integral of $\nabla \phi$ in the first term can be transformed to the surface integral of $\phi$ on the sphere surface $\partial B$ and the second term vanishes as $r \rightarrow \infty$. Further, integration on the sphere surface projected along the $z$-coordinate gives the drag

$$
F_{D}=-\rho \boldsymbol{k} \cdot \frac{d}{d t} \int_{V_{f}} \boldsymbol{u} d V=\rho \frac{d}{d t} \oint_{\partial B} \phi(\boldsymbol{k} \cdot \boldsymbol{n}) d S=-\frac{1}{2} \rho\left(\frac{4}{3} \pi R^{3}\right) \frac{d V_{s}}{d t} .
$$

Clearly, Eq. (A2) recovers the classical formula of the added mass force on a sphere moving with an unsteady acceleration where the added mass coefficient is $1 / 2$. This indicates that the second and fifth terms together are reduced to the added mass force when the flow is inviscid and irrotational. In general, the velocity field can be decomposed into the potential flow part and viscous flow part, i.e., $\boldsymbol{u}=\nabla \phi+\boldsymbol{u}_{v i s}$, where $\boldsymbol{u}_{v i s}$ is the velocity deviation from the potential flow due to fluid viscosity. Therefore, in addition to the added mass force, the second term in RHS of Eq. (2) as an inertial term naturally includes the effects of viscous flow phenomena. For example, in separated viscous flows over a stationary body where the added mass force is zero, the second term in RHS of Eq. (2) is purely induced by unsteady flow structures generated by some intrinsic flow instability mechanisms such as the Kelvin-Helmholtz instability and vortex shedding.

\section{APPENDIX B: CODE VALIDATIONS}

The numerical method and code used in this work have been validated previously by investigating various flows including decaying vortices, $2 \mathrm{D}$ flows around stationary and oscillating cylinders, 3D flows over a sphere and a low-aspect ratio flat plate. ${ }^{33}$ In this appendix, additional comparisons with the existing experimental and computational data are provided for the flows around a flat plate.

In the first case, the flows around a stationary flat plate at different AoAs are simulated, and the results are compared with the measurements of Cleaver et $a l^{41}$ In the experimental setting, a $0.04 c$ thick flat plate with the semicircular leading and trailing edges is fixed in a uniform upstream flow, where $c$ is the chord length of the flat plate. The Reynolds number based on the incoming velocity and chord length is $\operatorname{Re}_{c}=1.0 \times 10^{4}$. The computational domain is $[-8 c, 12 c] \times[-2.5 c$, $2.5 c]$. The minimum and maximum grid length are $\delta h / c=0.0025$ and $\delta h / c=0.02$, respectively. The AoA of the flat plat varies from $0^{\circ}$ to $20^{\circ}$. The time-averaged lift coefficient is shown in Figure 16 as a function of AoA. The time-averaged lift coefficient increases linearly with AoA for $A o A<10^{\circ}$, which is consistent with the prediction of the classical thin-airfoil theory. For $A o A>$ $10^{\circ}$, after the flow fully separates, the lift coefficient saturates and decreases. The computational results are consistent with the experimental data of Cleaver $e t a l .{ }^{41}$ and the numerical results of Vargas et al..$^{42}$ At $\operatorname{Re}_{c}=1.0 \times 10^{4}$, the time-averaged lift coefficient in $A o A=0^{\circ}-10^{\circ}$ still follows the classical thin-wing or thin-airfoil theory that predicts the lift slope of $2 \pi$.

In the second case, the flow around a translational and pitching flat plate in still water is simulated to validate the present numerical method in handling the highly unsteady flows over the moving boundaries. The numerical results will be compared with the experimental data of Sunada et al. ${ }^{43}$ who simulated the motion of an insect wing in hovering. The translational and pitching kinematics are given by

$$
h=h_{a} \sin (2 \pi f t), \quad \alpha=\alpha_{0}+\alpha_{1} \sin (2 \pi f t+\phi),
$$

where $h$ is the position of the rotational axis of the plate in the $-X$ direction, $\alpha$ is the angle between the flat plate and $-X$ direction, $f$ and $h_{a}$ are the frequency and amplitude of the translational motion, respectively, $\alpha_{0}$ and $\alpha_{1}$ are the time-averaged pitching angle and pitching amplitude, respectively, 


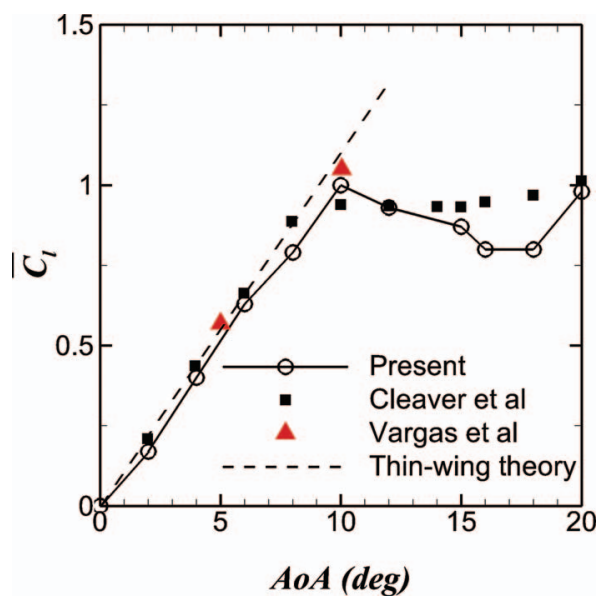

FIG. 16. Time-averaged lift coefficient of a flat plate as a function of AoA at $\operatorname{Re}_{c}=1.0 \times 10^{4}$.

$\phi$ is the phase difference between the translating and pitching motions. The rotation axis is at the quarter chord (c/4) from the leading edge. The parameter are selected as $h_{a}=1.4 c, \alpha_{0}=\pi / 2, \alpha_{1}=$ $\pi / 2$, and $\phi=\pi / 4$. The Reynolds number and the reduced frequency are given by

$$
\operatorname{Re}=\frac{2 \pi f h_{a} c}{v}=1000, \quad k=\frac{2 \pi f c}{2\left(2 \pi f h_{a}\right)}=\frac{c}{2 h_{a}}=0.35 .
$$

The flat plate has a thickness of $0.05 c$. The computational domain is $[-24 c, 24 c] \times[-24 c, 24 c]$. The minimum and maximum grid length are $\delta h / c=0.005$ and $\delta h / c=0.04$, respectively.

The unsteady force coefficients $C_{x}$ and $C_{z}$ in the $X$ direction (the translational direction) and the $Z$ direction (the normal direction to $X$ ) in a period after the initial effect disappeared are shown in Figure 17. The computational result of $C_{x}$ is basically consistent with the experimental data of Sunada et al ${ }^{43}$ although there is a phase shift. However, the computational value of $C_{z}$ is considerably smaller than the measurements although the time trace has a similar waveform with a phase shift. The differences may come from the measurement errors in the experiments. Sunada et al. ${ }^{44}$ pointed out that the measurements could suffer from the effect of the free surface wave and the 3D effect of the gap between the wing tip and the wall of the water tank. The $3 \mathrm{D}$ effect of the relatively small aspect ratio of the wing model may also lead to the difference between their measurements and the 2D computations. ${ }^{41}$

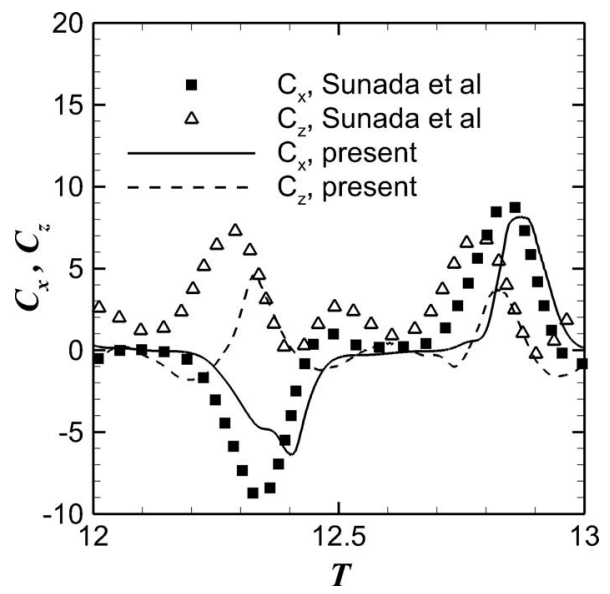

FIG. 17. The force coefficients $C_{x}$ and $C_{z}$ for the translational and pitching flat plate compared with the measurements of Sunada $\mathrm{et}$ al. 

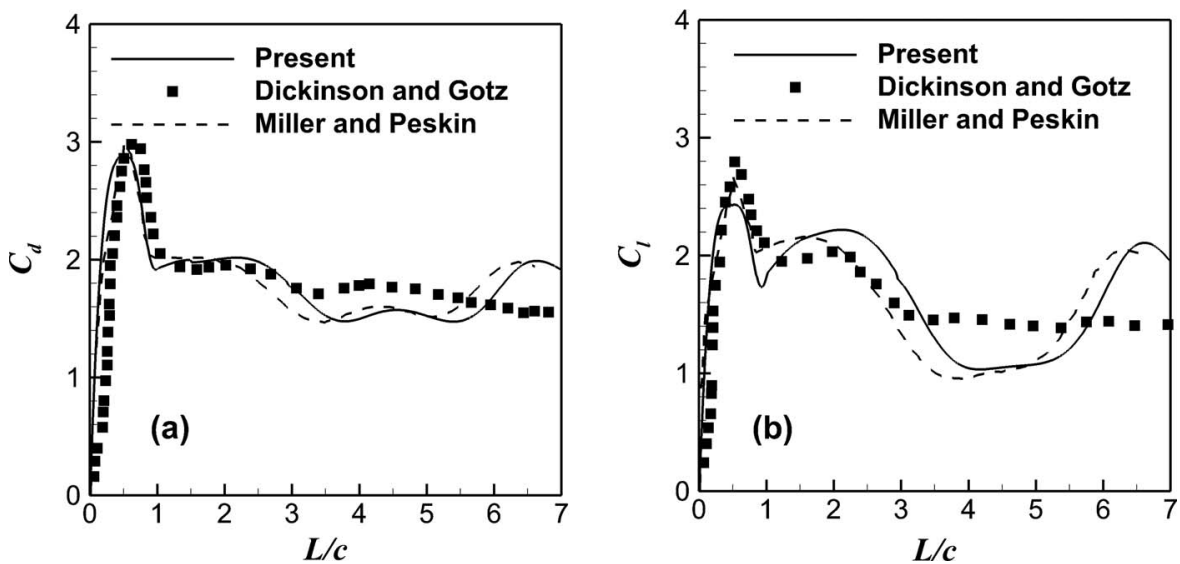

FIG. 18. The drag and lift coefficients of for a suddenly translating flat plat as a function of the normalized traveled distance: (a) the drag coefficient and (b) the lift coefficient.

In the third case, the flow around an impulsively starting and translating flat plate is investigated. The numerical parameters are set according to the experiments of Dickinson and Gotz. ${ }^{45}$ The flat plate with the thickness of $0.02 \mathrm{c}$ impulsively translates with a constant AoA in still water. The translational motion starts with a constant acceleration of $3.125\left(U^{2} / c\right)$ and then approaches to a fixed velocity $U$. The Reynolds number based on the fixed translational velocity and chord length is $\operatorname{Re}_{c}=192$. The computational domain in this case is $[-6 c, 14 c] \times[-4 c, 4 c]$. The minimum and maximum grid length are $\delta h / c=0.02$ and $\delta h / c=0.04$, respectively. The AoA of the flat plat is set at $45^{\circ}$ during the motion. The lift and drag coefficients $C_{l}$ and $C_{d}$ are plotted in Figure 18 as a function of the normalized traveled distance $L / c$. The computational results are consistent with the experimental data of Dickinson and Gotz ${ }^{45}$ and the numerical results of Miller and Peskin. ${ }^{46}$

${ }^{1}$ M. F. Platzer, K. D. Jones, J. Young, and J. C. S. Lai, "Flapping wing aerodynamics: Progress and challenges," AIAA J. 46(9), 2136-2149 (2008).

${ }^{2}$ W. Shyy, Y. Lian, J. Tang, D. Viieru, and H. Liu, Aerodynamics of Low Reynolds Number Flyers (Cambridge University Press, New York, 2008).

${ }^{3}$ G. R. Spedding, M. Rosen, and A. Hedenstrom, "A family of vortex wakes generated by a thrush nightingale in free flight in a wind tunnel over its entire natural range of flight speeds," J. Exp. Biol. 206(14), 2313-2344 (2003).

${ }^{4}$ A. Hedenstrom, L. C. Johansson, M. Wolf, R. von Busse, Y. Winter, and G. R. Spedding, "Bat flight generates complex aerodynamic tracks," Science 316(5826), 894-897 (2007).

${ }^{5}$ F. T. Muijres, L. C. Johansson, R. Barfield, M. Wolf, G. R. Spedding, and A. Hedenstrom, "Leading-edge vortex improves lift in slow-flying bats," Science 319(5867), 1250-1253 (2008).

${ }^{6}$ T. Y. Hubel, N. I. Hristov, S. M. Swartz, and K. S. Breuer, "Time-resolved wake structure and kinematics of bat flight," Exp. Fluids 46(5), 933-943 (2009).

${ }^{7}$ T. Y. Hubel, D. K. Riskin, S. M. Swartz, and K. S. Breuer, "Wake structure and wing kinematics: the flight of the lesser dog-faced fruit bat, cynopterus brachyotis," J. Exp. Biol. 213(20), 3427-3440 (2010).

${ }^{8}$ J. M. V. Rayner, "Vortex theory of animal flight 1. Vortex wake of a hovering animal," J. Fluid Mech. 91(APR), 697-730 (1979).

${ }^{9}$ J. M. V. Rayner, "Vortex theory of animal flight 2. Forward flight of birds," J. Fluid Mech. 91(APR), 731-763 (1979).

${ }^{10}$ C. P. Ellington, "The aerodynamics of hovering insect flight. V. A vortex theory," Phil. Trans. R. Soc. Lond. B 305(1122), 115-144 (1984).

${ }^{11}$ P. Watts, E. J. Mitchell, and S. M. Swartz, “A computational model for estimating the mechanics of horizontal flapping flight in bats: model description and validation,” J. Exp. Biol. 204(16), 2873-2898 (2001).

${ }^{12}$ T. L. Hedrick, J. R. Usherwood, and A. A. Biewener, "Low speed maneuvering flight of the rose-breasted cockatoo (eolophus roseicapillus). Ii. Inertial and aerodynamic reorientation,” J. Exp. Biol. 210(11), 1912-1924 (2007).

${ }^{13}$ S. P. Sane, "The aerodynamics of insect flight," J. Exp. Biol. 206(23), 4191-4208 (2003).

${ }^{14}$ S. A. Ansari, R. Zbikowski, and K. Knowles, "Aerodynamic modelling of insect-like flapping flight for micro air vehicles," Prog. Aerosp. Sci. 42(2), 129-172 (2006).

${ }^{15} \mathrm{Y}$. Yu, B. Tong, and H. Ma, "An analytic approach to theoretical modeling of highly unsteady viscous flow excited by wing flapping in small insects," Acta Mech. Sin. 19(6), 508-516 (2003).

${ }^{16}$ P. G. Saffman, Vortex Dynamics (Cambridge University Press, New York, 1992).

${ }^{17}$ C.-C. Chang, "Potential flow and forces for incompressible viscous flow," Proc. R. Soc. London, Ser. A 437(1901), 517-525 (1992). 
${ }^{18}$ M. S. Howe, "On unsteady surface forces, and sound produced by the normal chopping of a rectilinear vortex," J. Fluid Mech. 206, 131-153 (1989).

${ }^{19}$ J. Z. Wu and J. M. Wu, "Vorticity dynamics on boundaries," Adv. Appl. Mech. 32, 119-275 (1996).

${ }^{20}$ F. Noca, D. Shiels, and D. Jeon, "A comparison of methods for evaluating time-dependent fluid dynamic forces on bodies, using only velocity fields and their derivatives," J. Fluid Struct. 13(5), 551-578 (1999).

${ }^{21}$ J. Z. Wu, H. Y. Ma, and M. D. Zhou, Vorticity and Vortex Dynamics (Springer, Berlin, 2006).

${ }^{22}$ J. Z. Wu, X. Y. Lu, and L. X. Zhuang, "Integral force acting on a body due to local flow structures," J. Fluid Mech. 576, 265-286 (2007).

${ }^{23}$ C. Marongiu and R. Tognaccini, "Far-field analysis of the aerodynamic force by lamb vector integrals," AIAA J. 48(11), 2543-2555 (2010).

${ }^{24}$ L. M. Milne-Thomson, Theoretical Hydrodynamics, 5th ed. (Dover Publications, New York, 2011).

${ }^{25}$ J. D. Anderson, Fundamentals of Aerodynamics, 5th ed. (McGraw-Hill, New York, 2010).

${ }^{26}$ T. Liu and J. P. Sullivan, Pressure and Temperature Sensitive Paints (Springer, Berlin, 2004).

${ }^{27}$ T. Liu, J. Montefort, S. Woodiga, P. Merati, and L. Shen, "Global luminescent oil-film skin-friction meter," AIAA J. 46(2), 476-485 (2008).

${ }^{28}$ L. Quartapelle and M. Napolitano, "Force and moment in incompressible flows," AIAA J. 21(6), 911-912 (1983).

${ }^{29}$ T. von Karman and J. Burgers, "General Aerodynamic Theory - Perfect Fluids," in Aerodynamic Theory, edited by F. Durand (Dover Publications, New York 1963).

${ }^{30}$ J. C. Wu, "Theory for aerodynamic force and moment in viscous flows," AIAA J. 19(4), 432-441 (1981).

${ }^{31}$ J. C. Wu, Elements of Vorticity Aerodynamics (Tsinghua University Press, Beijing, 2005).

${ }^{32} \mathrm{~S}$. Schmitz and J.-J. Chattot, "Flow physics and stokes' theorem in wind turbine aerodynamics," Comput. Fluids 36(10), 1583-1587 (2007).

${ }^{33} \mathrm{~S}$. Z. Wang and X. Zhang, "An immersed boundary method based on discrete stream function formulation for two- and three-dimensional incompressible flows," J. Comput. Phys. 230(9), 3479-3499 (2011).

${ }^{34}$ K. Taira and T. I. M. Colonius, "Three-dimensional flows around low-aspect-ratio flat-plate wings at low Reynolds numbers," J. Fluid Mech. 623, 187 (2009).

${ }^{35}$ S. Yarusevych and M. S. H. Boutilier, "Vortex shedding of an airfoil at low Reynolds numbers," AIAA J. 49(10), 2221-2227 (2011).

${ }^{36}$ G. K. Taylor, R. L. Nudds, and A. L. R. Thomas, "Flying and swimming animals cruise at a strouhal number tuned for high power efficiency," Nature (London) 425(6959), 707-711 (2003).

${ }^{37}$ T. Theodorsen, "General theory of aero dynamic instability and the mechanism of flutter," NACA Technical Report No. 496, 1935.

${ }^{38}$ M. Visbal, "High-fidelity simulation of transitional flows past a plunging airfoil," AIAA Paper 2009-391, 2009.

${ }^{39}$ C. K. Kang, Y. S. Baik, L. Bernal, M. Ol, and W. Shyy, "Fluid dynamics of pitching and plunging airfoils of Reynolds number between $1 \times 10^{4}$ and $6 \times 10^{4}$," AIAA Paper 2009-536, 2009 .

${ }^{40}$ B. W. McCormick, Aerodynamics, Aeronautics, and Flight Mechanics (Wiley, 1995).

${ }^{41}$ D. J. Cleaver, Z. Wang, and I. Gursul, "Investigation of high-lift mechanisms for a flat-plate airfoil undergoing smallamplitude plunging oscillations," AIAA J. 51(4), 968-980 (2013).

${ }^{42}$ A. Vargas, R. Mittal, and H. Dong, "A computational study of the aerodynamic performance of a dragonfly wing section in gliding flight," Bioinspir. Biomim. 3(2), 026004 (2008).

${ }^{43}$ S. Sunada, K. Kawachi, A. Matsumoto, and A. Sakaguchi, "Unsteady forces on a two-dimensional wing in plunging and pitching motions," AIAA J. 39(7), 1230-1239 (2001).

${ }^{44}$ S. Sunada, T. Yasuda, K. Yasuda, and K. Kawachi, "Comparison of wing characteristics at an ultralow Reynolds number," J. Aircraft 39(2), 331-338 (2002).

${ }^{45}$ M. H. Dickinson and K. G. Gotz, “Unsteady aerodynamic performance of model wings at low Reynolds-numbers," J. Exp. Biol. 174, 45-64 (1993).

${ }^{46}$ L. A. Miller and C. S. Peskin, "When vortices stick: An aerodynamic transition in tiny insect flight,” J. Exp. Biol. 207(17), 3073-3088 (2004) 